\title{
Il-10 signaling reduces survival in mouse models of synucleinopathy
}

Samuel G. Cockey (D) ${ }^{1}$, Karen N. McFarland ${ }^{1,2,3,4}$, Emily J. Koller ${ }^{1,5}$, Mieu M. T. Brooks ${ }^{1,6}$, Elsa Gonzalez De La Cruz ${ }^{1}$, Pedro E. Cruz ${ }^{1,5}$, Carolina Ceballos-Diaz ${ }^{1}$, Awilda M. Rosario ${ }^{1}$, Yona R. Levites ${ }^{1,2,4,5}$, David R. Borchelt ${ }^{1,2,4,5}$, Todd E. Golde ${ }^{1,2,4,5}$, Benoit I. Giasson ${ }^{1,2,4,5}$ and Paramita Chakrabarty (iD) ${ }^{1,2,4,5 凶}$

Parkinson's disease (PD) and related synucleinopathies are characterized by chronic neuroinflammation leading to the premise that anti-inflammatory therapies could ameliorate synucleinopathy and associated sequelae. To test this idea, we used recombinant adeno-associated viruses (AAV) to express the anti-inflammatory cytokine, Interleukin (II)-10, in Line M83 transgenic mice that expresses the PD-associated A53T mutant human a-synuclein (aSyn). Contrary to our expectations, we observed that intraspinal II-10 expression initiated at birth upregulated microgliosis and led to early death in homozygous M83+/+ mice. We further observed that II-10 preconditioning led to reduced lifespan in the hemizygous M83+/- mice injected with preformed aSyn aggregates in hindlimb muscles. To determine the mechanistic basis for these adverse effects, we took advantage of the I87A variant II-10 (vll-10) that has predominantly immunosuppressive properties. Sustained intraspinal expression of vll-10 in preformed aSyn-aggregate seeded M83+/- mice resulted in earlier death, accelerated aSyn pathology, pronounced microgliosis, and increased apoptosis compared to control mice. AAV-vll-10 expression robustly induced p62 and neuronal LC3B accumulation in these mice, indicating that II-10 signaling mediated preconditioning of the neuraxis can potentially exacerbate aSyn accumulation through autophagy dysfunction in the neurons. Together, our data demonstrate unexpected adverse effects of both II-10 and its immunosuppressive variant, vll-10, in a mouse model of PD, highlighting the pleiotropic functions of immune mediators and their complex role in non-cell autonomous signaling in neurodegenerative proteinopathies.

npj Parkinson's Disease (2021)7:30; https://doi.org/10.1038/s41531-021-00169-8

\section{INTRODUCTION}

a-synucleinopathies are a heterogeneous group of disorders that are characterized by intracellular accumulation of a-synuclein (aSyn). These disorders, such as Parkinson's disease (PD), multiple systems atrophy (MSA), and dementia with Lewy body (DLB), are associated with robust neuroinflammation ${ }^{1,2}$. Genome-wide association studies and transcriptome analysis have uncovered increased genetic risk of $\mathrm{PD}$ in patients carrying specific variants of the major histocompatibility complex (MHC) class II genes and the LRRK2 gene, suggesting that immune pathways influence disease pathogenesis ${ }^{3-5}$. Several studies have reported increased inflammatory plasma cytokines as well as altered T-cell profiles in PD patients associated with specific inflammatory cytokines ${ }^{6,7}$. Preclinical modeling studies in aSyn transgenic mice have shown that aSyn activates immune cells by binding to Toll-like receptors and receptors on $\mathrm{T}$ cells $\mathrm{s}^{8,9}$. These studies confirmed that extracellular aSyn functions as damageassociated molecular patterns (DAMP), resembling pathogenassociated agents that trigger innate immunity ${ }^{10}$. Collectively, cohort analysis data, as well as experimental modeling studies, strongly suggest pathological synergism between chronic neuroinflammation and synuclein proteinopathy leading to the premise that neuroinflammation is a molecular driver in synucleinopathies ${ }^{11}$.

A major immuno-regulator during pathogen infections in the peripheral immune system is interleukin (II)-10 12 . II-10 modulates the activity of monocytes and macrophages leading to suppression of inflammatory cytokine expression and establishing anti-inflammatory conditions that foster tissue repair following pathogenic virulence.
Based on the hypothesis that chronic neuroinflammation underlies the etiology of synucleinopathy and extracellular aSyn has DAMP-like properties, anti-inflammatory mediators such as II-10 would be expected to ameliorate aSyn proteinopathy by dampening neuroinflammation. To test this hypothesis, we injected adeno-associated viruses (AAV) expressing II-10 into neonatal homozygous Line M83 $+/+$ mice or neonatal hemizygous M83+/- mice injected with preformed aSyn aggregates ${ }^{13-15}$. Contrary to our expectations, we found that II-10 preconditioning reduced lifespan in both mouse models, without altering the pathological burden of aSyn. As II-10 can have pleiotropic properties, we next used the anti-inflammatory variant, I87A variant (v)-II-10 16,17 , to fully characterize this unexpected outcome of II-10 signaling. We found that sustained expression of vll10 in hemizygous M83+/- mice seeded with preformed aSyn aggregates was also detrimental but in a manner distinct from II-10 and more consistent with accelerated aSyn pathology. We further identified neuronal autophagic dysfunction as a possible mechanism underlying the injurious outcome of vll-10 signaling in aSynaggregate-seeded mice. Together these studies document the unexpected adverse effects of II-10 preconditioning in mouse models of aSyn pathology.

\section{RESULTS}

II-10 increases microgliosis and accelerates death in homozygous $\mathbf{M 8 3}+I+$ mice

Recent evidence has established that a robust inflammatory milieu is associated with neurodegenerative phenotype in

\footnotetext{
${ }^{1}$ Center for Translational Research in Neurodegenerative Disease, University of Florida, Gainesville, FL, USA. ${ }^{2}$ Norman Fixel Institute for Neurological Diseases, University of Florida, Gainesville, FL, USA. ${ }^{3}$ Department of Neurology, University of Florida, Gainesville, FL, USA. ${ }^{4}$ McKnight Brain Institute, University of Florida, Gainesville, FL, USA. ${ }^{5}$ Department of Neuroscience, University of Florida, Gainesville, FL, USA. ${ }^{6}$ Present address: Department of Neuroscience, Mayo Clinic, Jacksonville, FL, USA. ${ }^{\bowtie}$ email: pchakrabarty@ufl.edu
} 
several proteinopathies, including Parkinson's disease (PD) ${ }^{11}$. Therefore, we wanted to examine whether suppressing inflammation would have a beneficial effect on proteinopathy and survival in the transgenic Line M83 mice that expresses the PDassociated A53T mutant human aSyn (SNCA). Since II-10 has been shown to attenuate Th1 type immune response and moderate macrophage/monocyte activation ${ }^{12}$, we hypothesized that II-10 expression would regulate inflammation leading to suppression of aSyn pathologies and extending lifespan in these mice. We used recombinant AAV serotype 1 to express mouse II-10 under the control of hybrid chicken $\beta$-actin promoter, which was injected in the lumbar segment of the spinal cord of neonatal mice as previously described ${ }^{18}$. For the initial dose-finding study, we tested three different doses of AAV-II-10 delivered into the lumbar spinal cord of nontransgenic (nonTG) mice on neonatal day P0 (Supplementary Fig. 1a-c). We found that injection of the highest dose $\left(1 \times 10^{10}\right.$ viral genomes) resulted in consistent levels of II-10 protein detectable in the soluble lysates of the spinal cord and CSF (Supplementary Fig. 1a, b), suggesting that the II-10 was being secreted following cellular production. Further experiments were conducted using this high dose. We did not find reproducibly detectable amounts of II-10 in the serum of these injected mice (Supplementary Fig. 1c). Injection of this highest dose of II-10 also resulted in increased astrocytosis in the brains of nonTG mice as revealed by GFAP immunoblotting and immunohistochemistry (Supplementary Fig. 1d-f).

To test the effect of II-10 signaling on synucleinopathy, we delivered AAV-II-10 and AAV-green fluorescent protein (GFP) into the spinal cords of neonatal homozygous $M 83+/+$ mice $^{13}$. We detected $7.8 \pm 2.6 \mathrm{ng} / \mathrm{ml}$ of II-10 protein in the soluble fraction of spinal cord lysates of AAV-II-10 injected mice compared to $0.46 \pm$ $0.4 \mathrm{ng} / \mathrm{ml}$ of II-10 protein in control mice injected with AAV-GFP ( $n$ $=3$ mice/group; $P<0.01$ ). As these mice aged, the II-10-expressing $\mathrm{M} 83+/+$ mice exhibited an abnormal phenotype marked by hunched posture, loss of body weight $(>20 \%)$, labored breathing, and unusually high instances of sudden death. Compared to the GFP-expressing control cohort that exhibited $\sim 20 \%$ death by 250 days of age, the median age of survival of II-10-expressing $\mathrm{M} 83+/+$ mice was 138 days (Fig. $1 \mathrm{a} ; P<0.0001$ ). We found that II-10 expression resulted in increased microgliosis (measured by $\mathrm{cd} 11 \mathrm{~b}$ immunoreactivity) in the cortex and midbrain areas relative to phenotypically matched AAV-GFP mice (Fig. 1b; cortex, $P<0.01$; midbrain, $P<0.05)$. Microgliosis was not significantly upregulated in either thoracic or lumbar segments of spinal cords of the II-10expressing $\mathrm{M} 83+/+$ mice (Fig. 1c). Astrocytosis (measured by GFAP immunoreactivity) was upregulated in the cortex of II-10expressing mice (Fig. 1d; $P<0.05$ ), while midbrain and spinal cord did not show significant changes compared to GFP-expressing control mice (Fig. 1d, e). We wanted to examine whether the early death triggered by II-10 was accompanied by the accelerated formation of aSyn inclusions in the CNS of M83+/+ mice. We used the pSer129-aSyn epitope-specific antibody to detect pathologic aSyn inclusions and the p62 antibody as a general marker of autophagy failure and protein aggregation in these tissues. Using the pSer129-aSyn-specific 81A antibody (Fig. 1f-h) or anti-p62 antibody (Fig. 1i-k), we did not observe any significant induction of aSyn pathology in the II-10-expressing mice compared to GFPexpressing controls, suggesting that II-10-mediated immune dysregulation can cause neurotoxicity and accelerated death independent of obvious proteinopathy abnormalities.

To understand the mechanism underlying II-10-induced early death, we used a custom NanoString codeset $^{19}$ to analyze gene expression changes in the thoracic spinal cords of II-10 and GFPexpressing $\mathrm{M} 83+/+$ mice that showed end-stage sickness and paralysis phenotype. We identified several genes that were upregulated in the II-10 mice-Fcgr2b, Ccl8, Chil3, Ccl5, Fcgr4, Ms4a6d, Abca1, Ptprc (Cd45), and Apoe-that broadly indicated an immune response associated with phagocytosis related pathways and Fc gamma receptor signaling pathways (Fig. 1I, m and Supplementary Table 1). II-10 was upregulated (log2 fold change = 13.49; $P=1.64 \times 10^{-51}$ ) in these mice but was omitted from the volcano plot in Fig. $1 \mathrm{l}$ as it skewed the plot (Fig. $1 \mathrm{l}$ and Supplementary Table 1). We were curious whether the accelerated death phenotype in II-10-expressing mice was mediated via transcriptional upregulation of the human SNCA transgene driven by mouse prion promoter. We found that II-10 expression did not change the levels of the SNCA transgene (log2 fold change $=$ $-0.16 ; P=0.55)$ in AAV-II-10-expressing $\mathrm{M} 83+/+$ mice relative to AAV-GFP-expressing M83+/+ mice (Supplementary Table 1). To establish the specific type of microglial profile associated with sustained II-10 expression in M83+/+ mice, we calculated gene expression scores corresponding to the prevalence of inflammatory phenotype (M1 phenotype), anti-inflammatory phenotype (M2 phenotype), and neurodegeneration-specific damage-associated microglial phenotype (DAM phenotype). These scores were imputed based on expression levels of a set of genes that uniquely characterize each of these phenotypes (reviewed in ref. ${ }^{20}$ ) (Fig. $1 n-p$ and Supplementary Table 2). Compared to GFPexpressing M83+/+ mice, we observed that II-10-expressing mice showed a transcriptional profile consistent with M2-type signature (Fig. 10; $P<0.01$ ). We did not observe any significant changes in the M1-type or DAM signature (Fig. 1n, p). We next compared the top altered genes in the II-10-expressing M83+/+ mice (normalized to GFP-expressing M83+/+ mice) (Supplementary Table 1) with paralyzed aged M $83+/+$ mice (normalized to nonTG mice) (Supplementary Table 3). A number of common genes were identified in both groups, such as Fcgr2b, Fcgr4, Ccl5, and Ms4a6d, that are involved in immune signaling (Supplementary Table 4). Such commonalities between these two groups suggest that II-10mediated immune dysfunction reinforced the neurodegenerative cascade in the M83+/ model.

\section{II-10 expression reduces lifespan in preformed aSyn fibril- seeded hemizygous $\mathbf{M 8 3}+I-$ mice}

Next, we examined whether II-10 preconditioning would modulate the induction and prion-like transmission of aSyn pathology in hemizygous M83+/- mice. In this model, preformed aSyn fibrils injected into the gastrocnemius muscle of 2-month-old M83 $+/-$ mice leads to progressive induction of aSyn pathology along the spinal axis and midbrain, with hindlimb paralysis occurring $\sim 4$ months after injection ${ }^{14,15}$.

Neonatal M83+/- mice were injected with AAV-II-10 or AAVGFP in the lumbar spinal cord and aged to 2 months when they were injected bilaterally in the hindlimb muscle with preformed aSyn fibrils (Fig. 2a). As the control for preformed aSyn fibril injection, mice were injected with the vehicle, PBS, in their hindlimb muscle. Biochemical analysis of the spinal cord lysates of mice revealed that at end stage, the aSyn-seeded AAV-II-10 and AAV-GFP-expressing M83+/- mice had $4.2 \pm 1.4 \mathrm{ng} / \mathrm{ml}$ and $1.6 \pm$ $0.06 \mathrm{ng} / \mathrm{ml}$ of $11-10$ protein, respectively $(n=5 \mathrm{mice} / \mathrm{group} ; P<$ $0.01)$. In the aSyn-seeded cohorts, we observed that II-10 reduced lifespan compared to the control group expressing GFP (Fig. 2a; median survival of II-10 mice $=112$ days and control mice $=$ 124.5 days post injection; $P<0.0001$ ). Non-seeded control mice (injected with vehicle PBS) expressing AAV-GFP or AAV-II-10 did not develop paralysis (Fig. 2a).

Using the custom neurodegeneration NanoString codeset, we examined gene expression changes in M83+/- mice expressing II-10. First, we examined the gene expression changes in PBS injected (non-seeded) M83+/- mice injected with AAV-II-10 or AAV-GFP. We found that II-10 expression leads to upregulation of various immune genes, such as complement factors, $\mathrm{Ccl} 8$, Fcgr2b, and Trem2 (Fig. 2b and Supplementary Table 5). II-10 was also robustly upregulated ( $\log 2$ fold change $=10.53 ; P=$ $5.89 \times 10^{-7}$ ) in these mice but was omitted from the volcano plot 

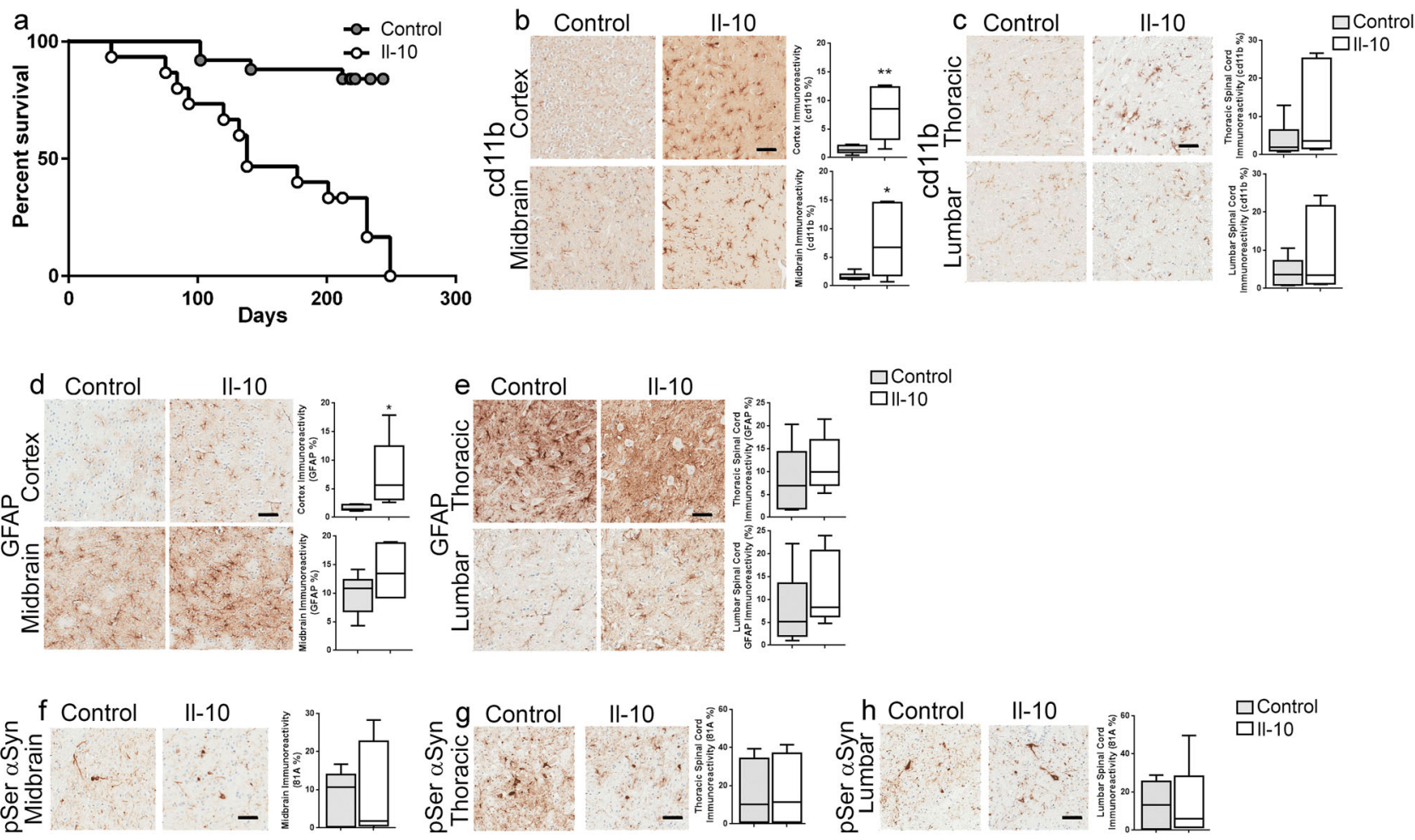

$\square$ Control
$\square \|-10$
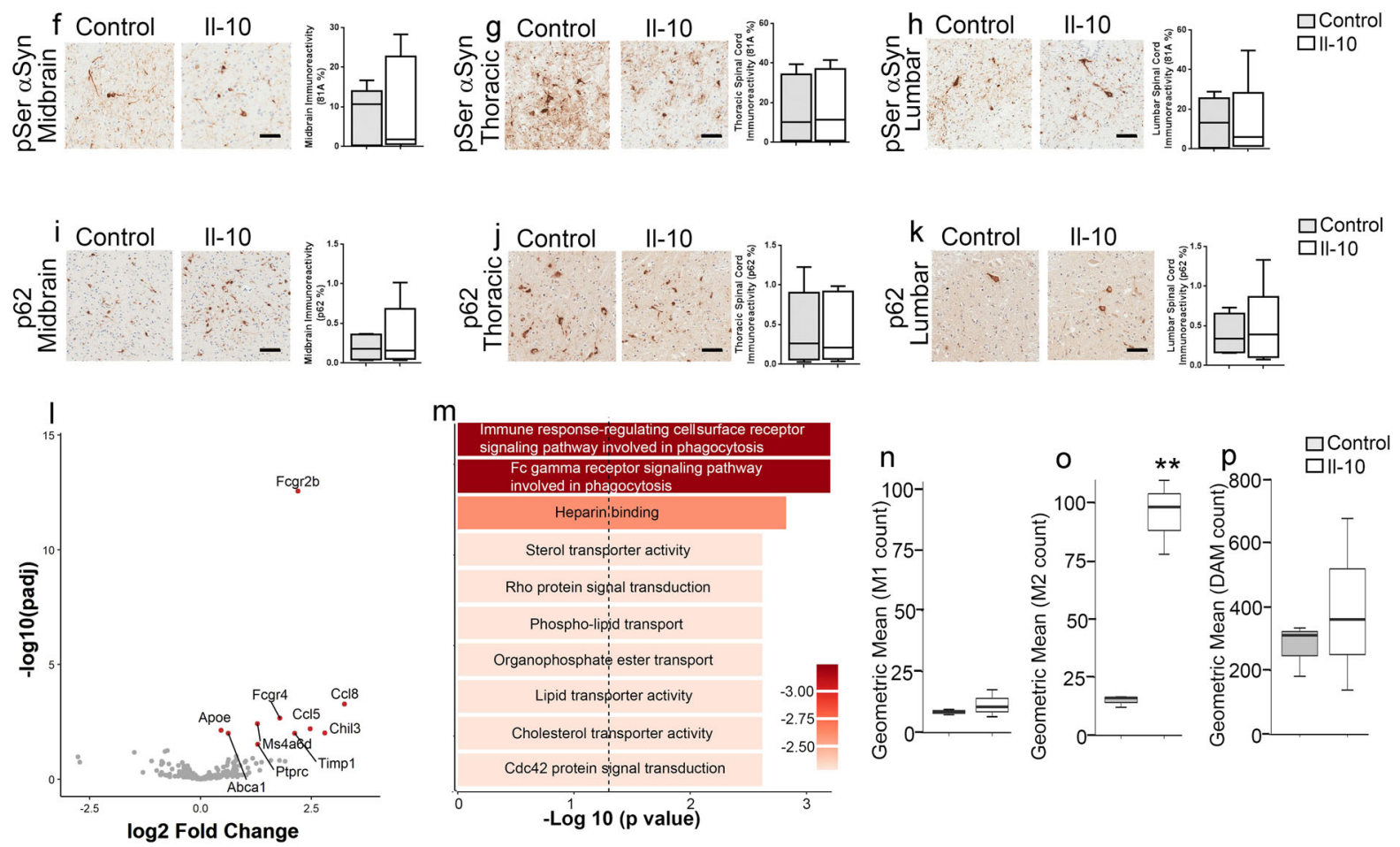

Fig. 1 Intraspinal II-10 expression accelerates death in homozygous M83 mice. a Intraspinal expression of AAV-II-10 leads to early mortality in $\mathrm{M} 83+/+$ mice. The median age of survival of II-10-expressing mice was 138 days, and the median age for the AAV-GFP-expressing mice was undefined (log-rank test, $P<0.0001, n=15-16$ mice/group). b, c Microgliosis (cd11b staining) in the brain (b, cortex and midbrain) and spinal cord (c, thoracic and lumbar segment) of M83+/+ mice. d, e Astrocytosis (GFAP staining) in the brain (d, cortex and midbrain) and spinal cord (e, thoracic and lumbar segment) of M83+/+ mice. $\mathbf{f}-\mathbf{k}$ Representative images from anti-81A (f-h) and anti-p62 (i-k) antibody-stained tissue. Immunostaining analysis is shown from the midbrain $(\mathbf{f}, \mathbf{i})$, the thoracic segment of the spinal cord $(\mathbf{g}$, $\mathbf{j})$, and the lumbar segment of the spinal cord (h, k). Scale bar: $50 \mu \mathrm{m} . n=6 \mathrm{mice} /$ group; two-tailed $t$ test. I-p Volcano plot (I) showing differential expression of genes in the thoracic spinal cords of II-10-expressing M83+/+ mice compared to GFP-expressing controls. Red dots, significantly changed genes, $P<0.05 ;$ gray dots, $P>0.05$. To avoid skewing the graph, the $x$ axis is limited to a range of -3 to +3 and as a result, Il-10 data is not shown (log2 FC $=13.49 ;$ Padj value $\left.=1.64 \times 10^{-51}\right)$. Gene ontology analysis of overrepresented categories using a list of significantly changed genes from panel I (Padj $<$ 0.05; dotted line) (m). $n=3$ mice/group. FDR $<0.05$. Padj $=P$ values adjusted for multiple comparisons. $\mathbf{n}-\mathbf{p}$ M1, M2-, and DAM-phenotype profile of microglia in II-10-expressing M83+/+ mice compared to GFP-expressing M83+/+ control mice. ${ }^{* *} P<0.01$. $n=3$ mice/group. In the box plot, the whiskers extend from the minimum to maximum values, with the midline representing the median. 
a
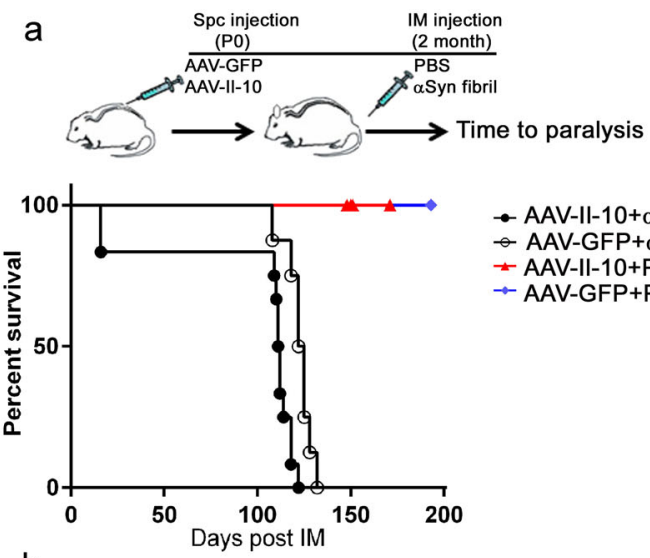

- AAV-II-10+ $\alpha$ Syn $(n=12)$

AAV-GFP+ $\alpha \operatorname{Syn}(n=18)$

- AAV-II-10+PBS $(n=14)$

$\rightarrow$ AAV-GFP+PBS $(n=10)$

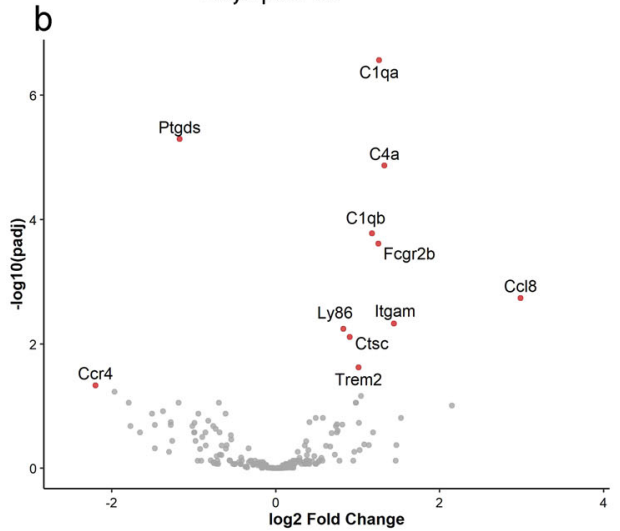

C
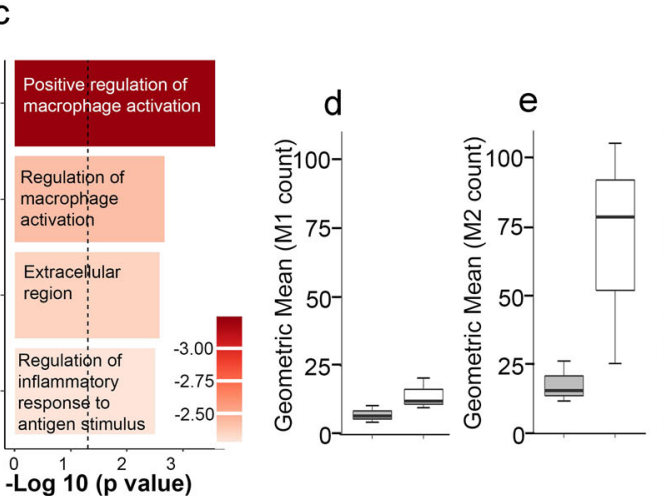

$\square$ AAV-GFP + PBS IM

$\square$ AAV-II-10 + PBS IM
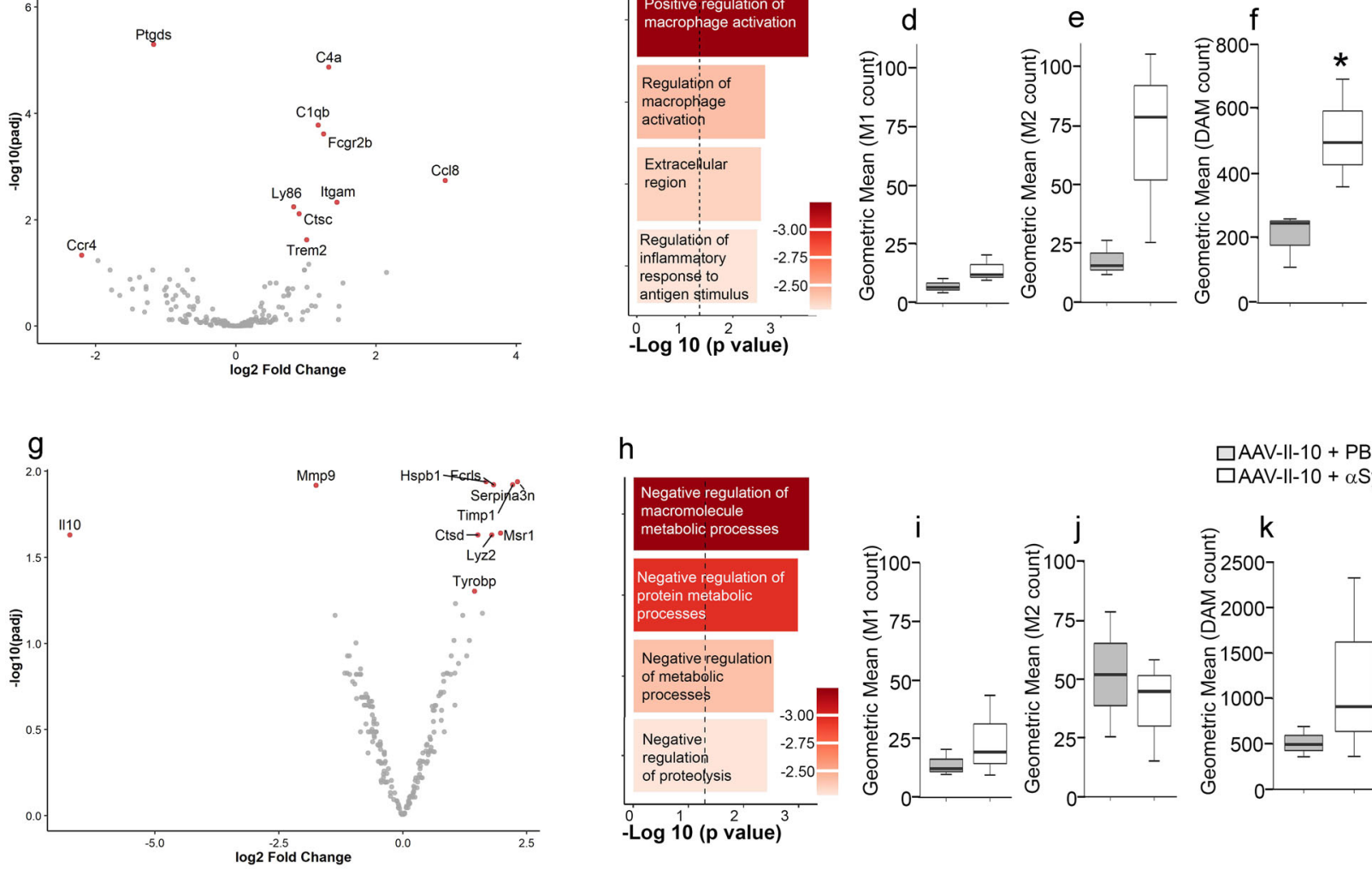

h
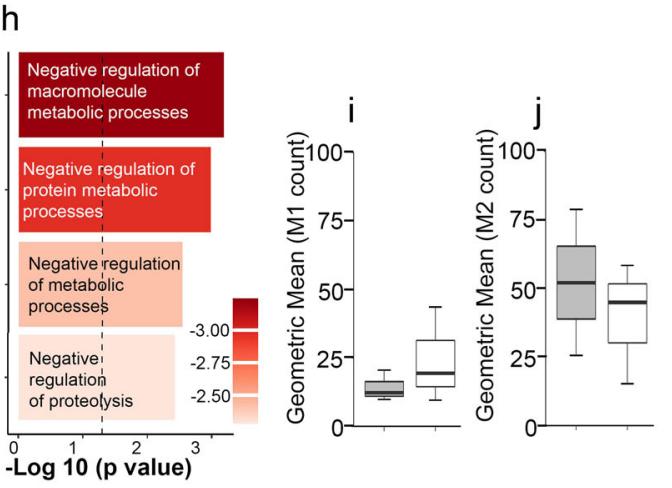

口AAV-II-10 + PBS IM

$\square$ AAV-II-10 + $\alpha$ Syn IM
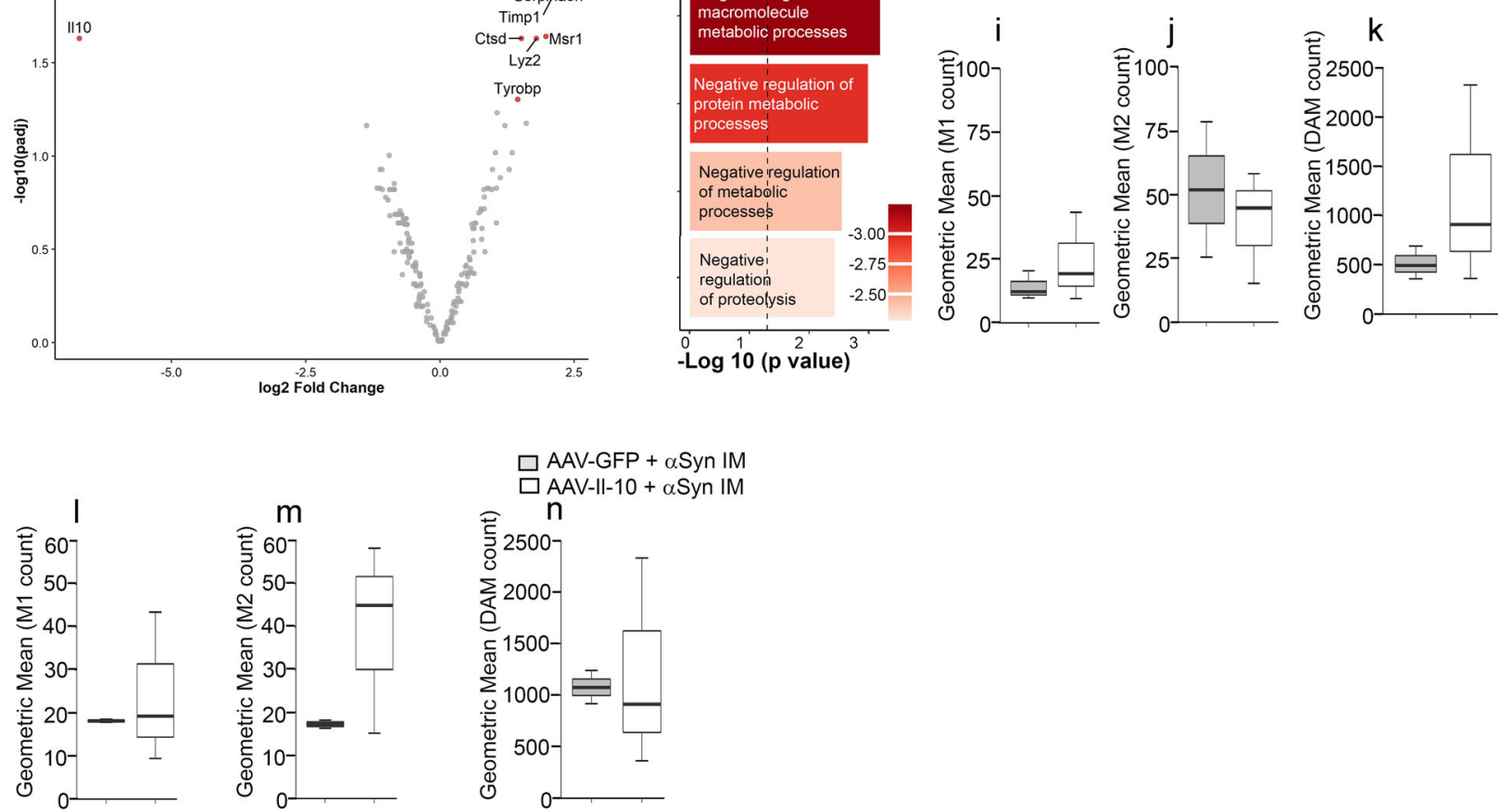

in Fig. 2b as it skewed the plot (Fig. 2b and Supplementary Table 5). GO pathway analysis indicates that II-10 expression induced robust inflammatory signaling characteristic of macrophage activation and presence of an antigenic stimulus (Fig. 2c). Neither M1-type nor M2-type gene expression signatures were significantly enhanced in these mice, though there was a suggestive trend in the $M 2$ signature (Fig. $2 \mathrm{~d}$, e; $P=0.32$ for M2). Interestingly, we observed that II-10 expression led to induction of DAM signature (Fig. 2f; $P<0.05$ ), primarily driven by increased expression of Trem2. 
Fig. 2 Intraspinal expression of AAV-II-10 leads to accelerated mortality in aSyn-aggregate-seeded hemizygous M83 $+I-$ mice. a Schematic summarizing the experimental procedure (top panel). M83+/- neonates were injected intraspinally (SpC) with AAV-II-10 or AAVGFP (control). Mice were aged to 2 months and injected intramuscularly (IM) with preformed $\alpha$ Syn fibrils or PBS (vehicle control). Mice were aged to paralysis. The median age of survival of $\alpha$ Syn-seeded II-10 or GFP-expressing mice was 112 days and 124.5 days post $\alpha$ Syn seeding respectively (log-rank test, $P<0.001, n=10-18 /$ group). $\mathbf{b}-\mathbf{n}$ NanoString analysis of the $\alpha$ Syn or vehicle (PBS)-seeded thoracic spinal cords of M83+/- mice expressing II-10 or GFP. b, c Volcano plot of differentially expressed genes (b) and gene ontology pathway analysis of overrepresented functional categories (c) accompanying II-10 overexpression in M83+/- spinal cords (Padj< 0.05 ; dotted line). To avoid skewing the volcano plot, the $x$ axis is limited to a range of -2.5 to +4 and as a result, II-10 data is not plotted (log $2 \mathrm{FC}=10.537 ;$ Padj value $=$ 5.9e-07). d-f M1-, M2-, and DAM-type microglial profiling in II-10-expressing M83+/- mice. Two-tailed $t$ test, $* P<0.05$. g, h Volcano plot of differentially expressed genes $(\mathbf{g})$ and gene ontology pathway analysis of overrepresented functional categories (h; Padj $<0.05$, dotted line) in $\alpha$ Syn-seeded II-10-expressing M83+/- mice vs vehicle-seeded II-10-expressing M83+/- mice. i-k M1-, M2-, and DAM-type microglial profiling in II-10-expressing M83+/- mice in the presence or absence of intramuscular (IM) $\alpha$ Syn seeding. I-n M1-, M2-, and DAM-type microglial profiling in II-10 vs GFP-expressing $\alpha$ Syn-seeded M83+/- mice. $n=3$ mice/group. Red dots significantly changed genes, $P<0.05$ and gray dots, $P>0.05$ in volcano plots after adjusting for multiple testing and FDR $<0.05$. In the box plot, the whiskers extend from the minimum to maximum values, with the midline representing the median.

Next, we compared the gene expression changes in II-10expressing $\mathrm{M} 83+\mathrm{I}-$ mice that had been seeded with preformed aSyn aggregates relative to II-10-expressing non-seeded (PBS injected) M83+/- mice (Supplementary Table 6). Here, we found that aSyn seeding in the presence of II-10 results in an exacerbated inflammatory milieu characterized by increased Lyz2, Msr1, Ctsd, and Tyrobp (Fig. $2 \mathrm{~g}$ and Supplementary Table 6). Overall, GO pathway analysis indicated reduced metabolic processes at different cellular and physiologic levels, indicative of a degenerative signature (Fig. 2h). There were no significant changes in $\mathrm{M} 1-, \mathrm{M}_{2}-$, or DAM-type profiles in the aSyn-seeded mice compared to mice injected with PBS vehicle control (Fig. 2i-k).

When we compared the DEG in aSyn-seeded M83+/- mice expressing II-10 to aSyn-seeded M83+/- mice expressing GFP at end stage, we observed that both the DEGs were extremely similar to each other such that there were no differentially expressed genes that could be identified from this comparison (Supplementary Table 7). Consistent with this observation, microglial profiling analysis did not show significant levels of difference between the two groups (Fig. 2l-n). To understand this neurodegenerative cascade, we performed an additional comparison. There were ten genes that were significantly altered in response to aSyn seeding in II-10-expressing mice (II-10-expressing aSyn-seeded mice normalized to II-10-expressing non-seeded mice) (Fig. $2 \mathrm{~g}$ and Supplementary Table 6). When we compared this list with genes altered in aSyn-seeded naive M83+/- mice normalized to nonseeded mice (Supplementary Table 8), we found that the differentially expressed genes (except for II-10) were represented in both the cases (Supplementary Table 9). The similarities in the list of altered genes indicate that the cellular pathways affected by II-10 preconditioning synergized with the normally ongoing neurodegenerative cascade in the aSyn-seeded M83+/- mice.

\section{An immunosuppressive variant of cellular II-10 reduces lifespan in preformed aSyn fibril-seeded M83 $+I-$ mice}

II-10 has pleiotropic properties-in addition to its wellrecognized immunosuppressive properties, it also has immunostimulatory functions ${ }^{21}$. For example, II-10 can exert stimulatory effects on lymphocytes, murine thymocytes, and murine mast cells ${ }^{22,23}$. Indeed, our gene expression data identified several genes indicative of an activated immune signature, such as Ccl8, Ly86, and Itgam, in II-10-expressing mice (Fig. 2b). In order to understand whether the unexpected detrimental phenotype in II-10-expressing $\mathrm{M} 83+/$ - mice was related to the immunosuppressive or immunostimulatory function of II-10, we decided to use a variant II-10 (vll-10) that lacks immunostimulatory function ${ }^{16,17}$.

Some viruses have appropriated the immunosuppressive properties of II-10 by expressing close homologs that could be beneficial in preserving viral pathogenesis through ineffective

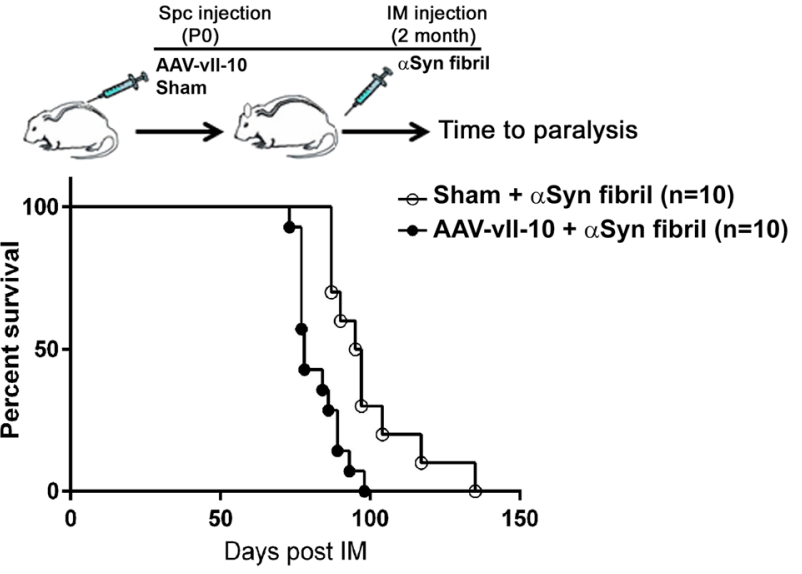

Fig. 3 Intraspinal expression of AAV-vIl-10 leads to early mortality in aSyn-seeded $\mathbf{M 8 3}+I-$ mice. M83 $+/-$ neonates were intraspinally injected with AAV-vll-10 or sham injected, aged to 2 months, and then injected with $\alpha$ Syn fibril in hindlimb muscles at 2 months of age. The median age of survival of vll-10-expressing mice was 78 days post $\alpha$ Syn fibril injection, while the median age of sham injected mice was 96 days (log-rank test, $P<0.0019, n=10$ mice/group).

host immune response ${ }^{17}$. One such homolog, produced by the Epstein Barr Virus and called $B C R F-l$, encodes a single point mutation in cellular II-10 (I87A II-10), referred to as vll-10 ${ }^{16}$. This vll10 has lost the stimulatory activities of cellular II-10 while retaining its immunosuppressive properties. In the next experiment, we delivered AAV-vll-10 into neonatal M83+/- mice, aged these to 2 months, seeded with preformed aSyn fibrils in the hindlimb muscle and aged them to paralysis. Analysis of the RIPA-soluble spinal cord lysates showed that the vll-10 levels in the AAV-vll-10 and control mice were $5.08 \pm 0.91 \mathrm{pg} / \mathrm{ml}$ and $0.47 \pm 0.13 \mathrm{pg} / \mathrm{ml}$, respectively ( $n=3$ mice; $P=0.007$ ). In this cohort of aSyn-seeded mice, we observed that vll-10 expression resulted in an accelerated paralysis phenotype (Fig. 3; $P<0.0019$ ). This data shows that an anti-inflammatory variant of $\mathrm{I}-10$ robustly reduces survival in aSyn-seeded M83+/- mice.

\section{vll-10 expression exacerbates microgliosis in preformed aSyn fibril-seeded M83 + I- mice}

We next conducted an extensive neuropathological analysis to characterize how II-10 and vll-10 expression modulate the induction and transmission of the aSyn pathology in hemizygous M83+/mice. We examined microgliosis using $\mathrm{cd} 11 \mathrm{~b}$ antibody in the spinal cords (Fig. 4a-d) and brains (Fig. 4e-h) of aSyn-seeded M83+/- 

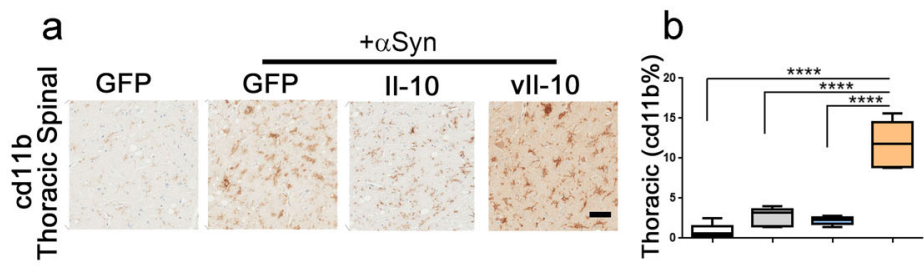

ㅁ GFP

$\square \mathrm{GFP}+\alpha \mathrm{Syn}$

ㅁII-10+ + Syn

$\square$ vll-10+ + Syn
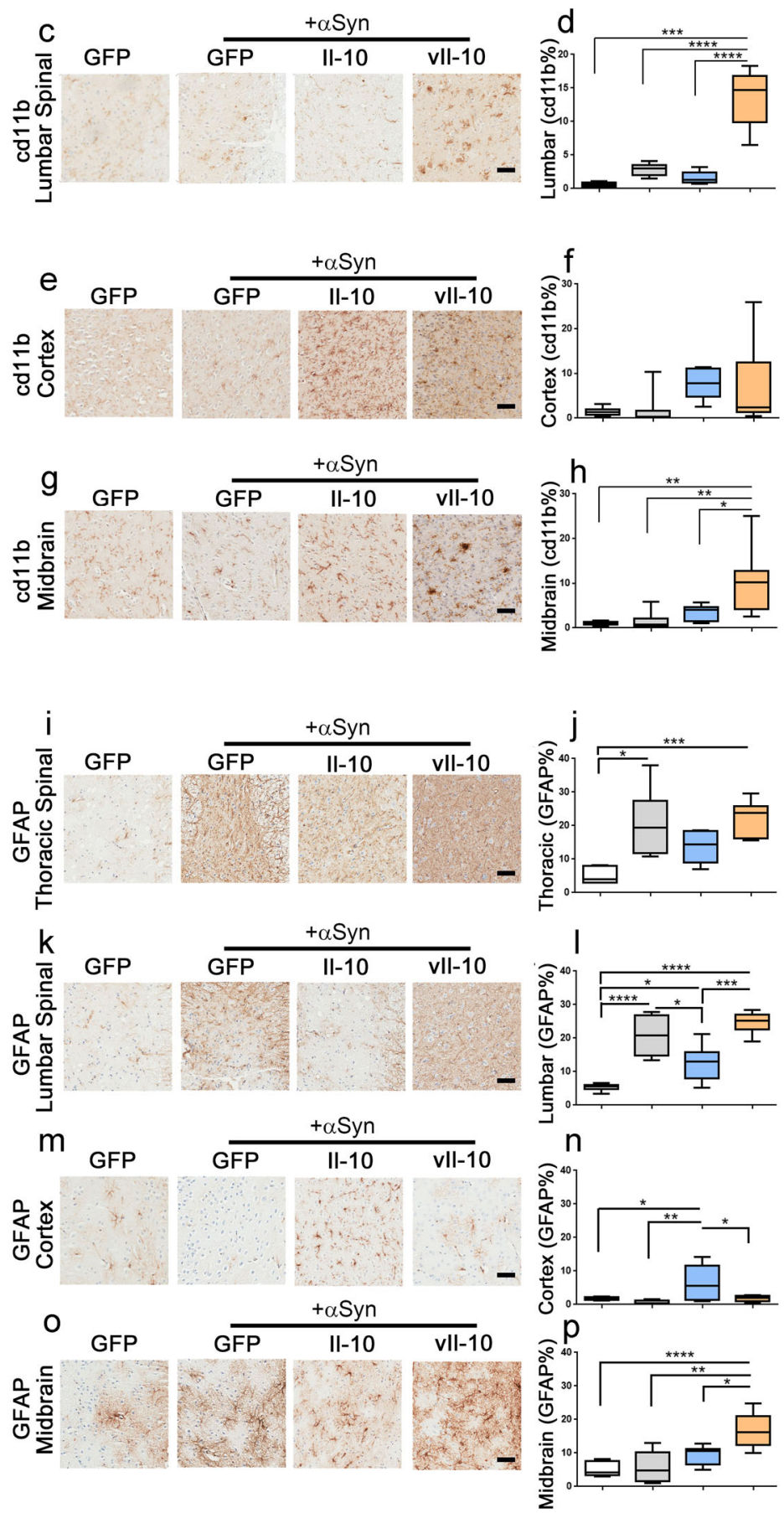

mice. Contrary to our expectation that vll-10 would dampen gliosis, we observed robust microgliosis in vll-10-expressing aSyn-seeded mice in both thoracic and lumbar segments of the spinal cord compared to control GFP-expressing PBS-injected mice, GFPexpressing aSyn-seeded mice or II-10-expressing aSyn-seeded mice
(Fig. 4a, b, $P<0.0001$ against all groups in the thoracic segment; Fig. 4c, d, $P<0.001$ against control and $P<0.0001$ against aSynseeded mice in the lumbar segment). While vll-10 expression also enhanced microgliosis in the midbrain area (Fig. $4 \mathrm{~g}, \mathrm{~h} ; P<0.05$ compared to II-10-expressing aSyn-seeded mice and $P<0.01$ 
Fig. 4 Intraspinal vIl-10 expression induces microgliosis but not astrogliosis in spinal cords of aSyn fibril-seeded hemizygous M83 $+/$ mice. Neonatal M83+/- mice were injected with AAV-GFP, AAV-II-10, or AAV-vIl-10 and were aged to 2 months when they were injected with preformed $\alpha$ Syn fibrils in the muscle. PBS (vehicle) injection was the control for $\alpha$ Syn fibril injection and is represented by the immunohistochemistry panel on the far left of each panel. a-h Representative $\mathrm{cd} 11 \mathrm{~b}$ staining patterns and immunostaining analysis (\% immunoreactivity burden) for microgliosis in thoracic segment of the spinal cord (a, b), lumbar segment of the spinal cord (c, d), cortex (e, f), and midbrain $(\mathbf{g}, \mathbf{h})$. i-p Representative GFAP staining and immunostaining analysis (\% immunoreactivity burden) for astrogliosis in thoracic segment of the spinal cord (i, $\mathbf{j})$, lumbar segment of the spinal cord $(\mathbf{k}, \mathbf{I})$, cortex $(\mathbf{m}, \mathbf{n})$, and midbrain $(\mathbf{o}, \mathbf{p})$. The whiskers in the box plot extend from the minimum to maximum values, with the midline representing the median. $n=6-7$ mice/group; one-way ANOVA; $* * * * P<0.0001$; ${ }^{* * *} P<0.001 ;{ }^{* *} P<0.01 ;{ }^{*} P<0.05$. Scale bar, $50 \mu \mathrm{m}$.

compared to GFP-expressing aSyn-seeded mice), there was no difference observed in the cortex relative to other mouse cohorts (Fig. 4e, f). We did not observe increased microglia numbers in the II-10-expressing aSyn-seeded mice relative to control aSyn-seeded mice in the spinal cord and the brain (Fig. 4a-h).

\section{II-10 expression suppresses astrocytosis in spinal cords of aSyn-seeded M83+I- mice}

We observed a complex pattern of astrocytosis in the aSyn-seeded hemizygous M83+/- mice (Fig. 4i-p). GFAP immunostaining in the thoracic and lumbar spinal cord showed that compared to PBS injected GFP-expressing hemizygous M83+/- mice, aSyn seeding induced astrocytosis in GFP-expressing mice as expected (Fig. 4i-l; $P<0.05$ in the thoracic segment and $P<0.0001$ in the lumbar segment). Within the aSyn-seeded cohorts, compared to GFP or vIL-10-expressing mice, expression of II-10 reduced astrocytosis in the lumbar spinal cord (Fig. 4k, l; $P<0.05$ against GFP and $P<$ 0.001 against vll-10), and there was a suggestive lowering trend in the thoracic spinal cord (Fig. $4 \mathrm{i}, \mathrm{j}$ ). In general, we observed that vll10 did not induce astrocytic proliferation in the spinal cord compared to the GFP-expressing mice that were seeded with preformed fibrils (Fig. 4j, I). In the brain, aSyn seeding, by itself, did not significantly alter astrocytosis in GFP-expressing mice (Fig. $4 \mathrm{~m}-\mathrm{p}$ ). In the cortex, the II-10-expressing mice had a higher astroglial burden compared to all other groups examined including vll-10-expressing mice (Fig. $4 \mathrm{~m}, \mathrm{n} ; P<0.05$ relative to vll-10 and $P<0.01$ relative to control seeded mice), consistent with our data in non-seeded homozygous M83+/+ mice (Fig. 1d). In the midbrains of mice seeded with preformed fibrils, we observed that vll-10 expression caused the highest level of astrocyte proliferation compared to non-seeded (PBS-injected) and aSynseeded control mice (Fig. 4o, p; $P<0.0001$ against non-seeded mice; $P<0.01$ and $P<0.05$ against seeded GFP-expressing and II-10-expressing mice respectively). This finding suggests that there is a selective vulnerability of astrocytes in different areas of the neuraxis to II-10- and vll-10-mediated signaling.

\section{vll-10 worsens pathological pSer129-aSyn inclusion pathology and increases aSyn levels}

As both II-10 and vll-10 caused accelerated paralysis in hemizygous M83+/- mice seeded with preformed aSyn fibrils, we next examined if this phenotype correlated with exacerbated aSyn proteinopathy. Consistent with our previous data ${ }^{14,15}$, injection of preformed aSyn fibrils in the gastrocnemius muscle of adult hemizygous M83+/- mice expressing AAV-GFP induced pSer129-aSyn reactive intracellular inclusions in the thoracic spinal cord, lumbar spinal cord, and midbrain compared to GFPexpressing non-seeded mice (Fig. 5a-f; $P<0.05$ ). These aSyn inclusions resemble Lewy bodies (LB) and were not observed in control GFP-expressing mice injected with the vehicle PBS (Fig. 5a, C, e). Expression of II-10 did not alter the levels of pSer129-aSyn immunoreactive LB-type pathology in the spinal cord or brain, compared to GFP-expressing aSyn-seeded mice (Fig. 5b, d, f). On the other hand, intraspinal vll-10 expression dramatically increased aSyn inclusion pathology in the thoracic and lumbar spinal cords compared to GFP-expressing and II-10-expressing seeded mice (Fig. 5b, d; $P<0.0001$ in thoracic; $P<0.001$ and $P<$ 0.0001 in lumbar segment respectively). In the midbrains of aSynseeded mice, the aSyn pathology burden was similar between all three cohorts (Fig. 5f).

The role of soluble aSyn in synucleinopathies is unclear. While features like LBs, that are characterized by clusters of aggregated aSyn inside neurons, are clearly neurotoxic, some studies have indicated that even modest increases in soluble aSyn protein levels can lead to cellular dysfunction ${ }^{24}$. Indeed, the early age of disease onset and disease progression severity in patients with duplication of wild-type aSyn loci indicates that simple intracellular accumulation of aSyn protein may have a profound pathogenic effect ${ }^{25}$. To investigate whether II-10-associated signaling pathways affect the accumulation of total aSyn levels, we used an antibody specific to the $C$ terminus of human aSyn, antibody 15-4E7, that was characterized previously in our lab ${ }^{26}$. Compared to both GFP and II-10-expressing aSyn-seeded mice, we observed that vll-10-expressing mice showed higher levels of 15-4E7-immunoreactive intracellular aSyn staining in both the thoracic and lumbar segments of the spinal cord (Fig. $5 \mathrm{~g}-\mathrm{j} ; P<$ 0.05 relative to $I 1-10$ and $P<0.01$ relative to GFP for the thoracic segment; $P<0.01$ relative to $\mathrm{II}-10$ and $P<0.001$ relative to GFP for the lumbar segment). Most of these stained structures were intracellular with some degree of parenchymal staining visible (Fig. 5g, i, k). Increased parenchymal staining with 15-4E7 antibody in the midbrain of vll-10-expressing mice compared to GFP-expressing mice $(P<0.0001)$ and $I I-10$-expressing mice $(P<0.001)$ indicates dramatic accumulation of extracellular aSyn in this brain area (Fig. $5 \mathrm{k}, \mathrm{I}$ ).

\section{vIl-10 induces cell death in spinal cords of aSyn fibril-seeded M83 + I- mice}

We wanted to examine whether II-10-mediated signaling pathways resulted in cell death in the brain and spinal cord. Since peripheral aSyn seeding induced midbrain aSyn pathology in our mice, we first quantified whether this led to a loss in dopaminergic neurons in these mice. Using tyrosine hydroxylase (TH) as the marker of dopaminergic cell bodies in the substantia nigra, we did not observe dopaminergic cell loss in any of our aSyn-seeded mouse cohorts compared to the control mice receiving vehicle injection (Fig. 6a, b).

We also assessed the effects of the II-10 signaling pathways on cell death in the spinal cords of M83+/- mice (Fig. $6 \mathrm{c}-\mathrm{f}$ ). We used two different reagents to investigate cell death in these mice. Fluoro-Jade $\mathrm{C}$ is an anionic dye used to detect degenerating or necrotic neurons and processes, irrespective of the type of death pathway involved ${ }^{27}$. Histochemical staining revealed increased Fluoro-Jade $\mathrm{C}$ punctate staining in the lumbar spinal cords of seeded vll-10-expressing mice compared to control GFPexpressing mice or II-10-expressing mice (Fig. $6 c$, d; $P<0.0001$ ). Such punctate staining is consistent with dying dendritic or axonal terminals. We further probed for activated caspase 3 to detect apoptotic cells in the lumbar spinal cords of seeded M83+/- mice (Fig. 6e, f). We found sparse activated caspase 3 immunopositive cells in the gray matter of the spinal cord as well as the spinal 

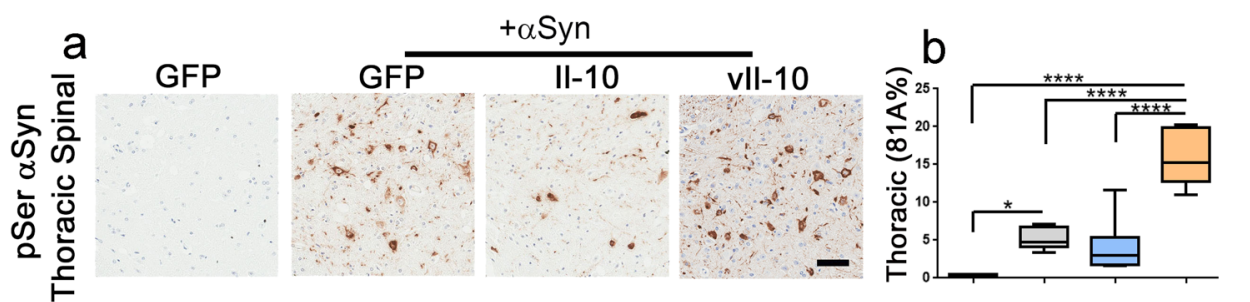

口GFP

GFP $+\alpha$ Syn

口II-10+ + Syn

$\square$ vll-10+ + Syn
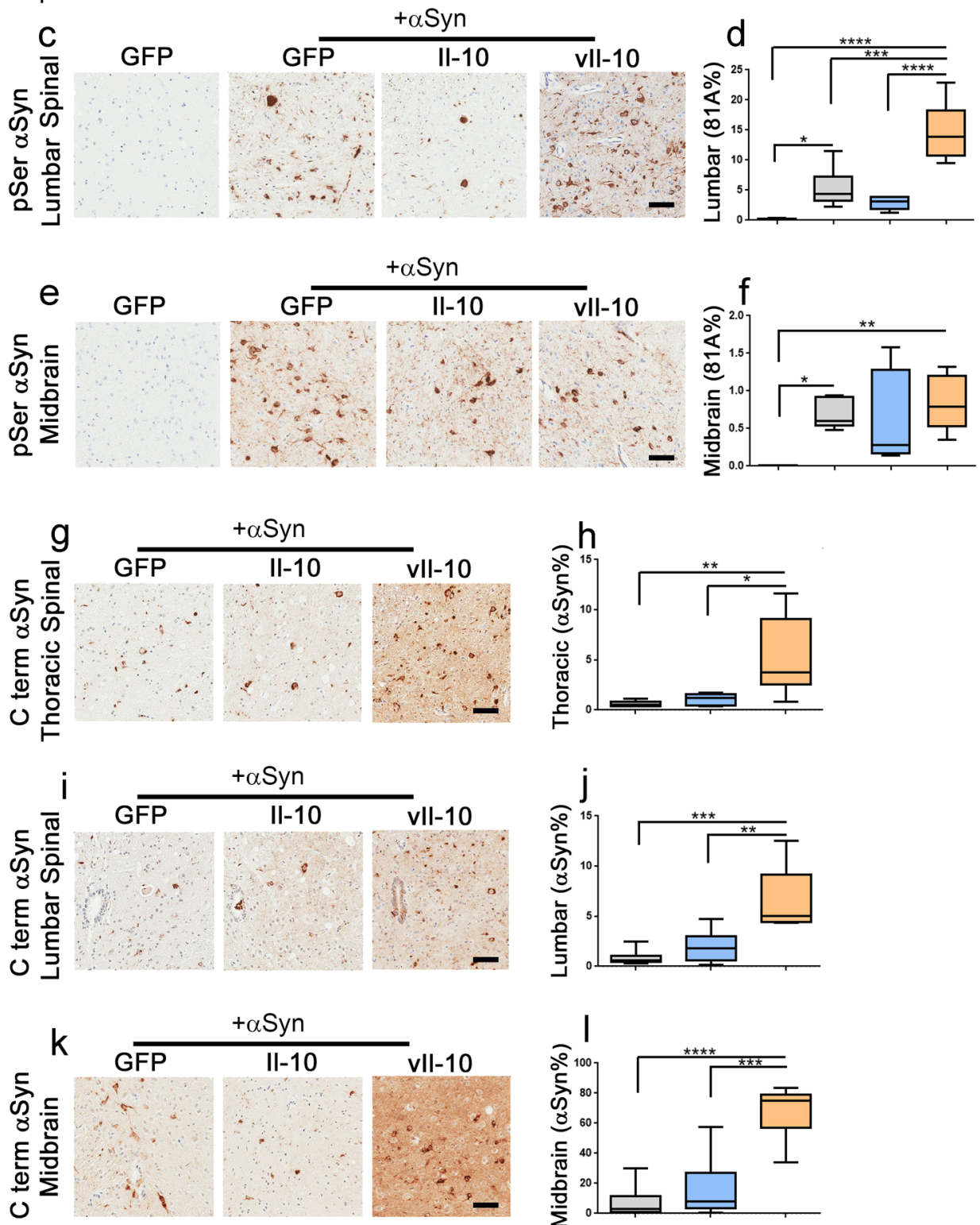

Fig. 5 vll-10 induces pathological aSyn inclusions and parenchymal aSyn accumulation in aSyn fibril-seeded hemizygous M83 $+I-$ mice. Neonatal M83+/- mice were injected with AAV-GFP, II-10, or vll-10 and were aged to 2 months when they were injected with $\alpha$ Syn fibrils in the muscle. PBS (vehicle) injection was the control for $\alpha$ Syn fibril injection and is represented by the immunohistochemistry panel on the far left (a, $\mathbf{c}, \mathbf{e})$. Representative images and \% immunoreactivity burden analysis of $81 \mathrm{~A}$ antibody (specific to pSer 129- $\alpha$ Syn epitope) stained tissue from the thoracic and lumbar segment of the spinal cord (a-d) and midbrain (e, f). Representative images and \% immunoreactivity burden analysis from 15-4E7 antibody (specific to the C terminus of $\alpha$ Syn) stained spinal cord ( $\mathbf{g}-\mathbf{j}$ ) and midbrain (k, I). In the box plot, the whiskers extend from the minimum to maximum values, with the midline representing the median. $n=5-7$ mice/group; one-way ANOVA; $* * * * P<$ $0.0001 ;{ }^{* * *} P<0.001 ;{ }^{* *} P<0.01 ;{ }^{*} P<0.05$. Scale bar, $50 \mu \mathrm{m}$.

ganglia of vll-10-expressing aSyn-seeded M83+/- mice (Fig. 6e, f; $P<0.0001)$, albeit to a lesser extent than observed with FluoroJade $C$ technique. None of the other aSyn-seeded mice demonstrated any detectable Fluoro-Jade $\mathrm{C}$ or activated caspase 3 reactivity (Fig. $6 \mathrm{~d}, \mathrm{f}$ ). vll-10 increases autophagy dysfunction in aSyn fibril-seeded M83+I- mice

We wanted to explore whether the increased parenchymal accumulation of soluble or cell-free aSyn, as well as intracellular inclusion pathology, could be due to impaired autophagic 


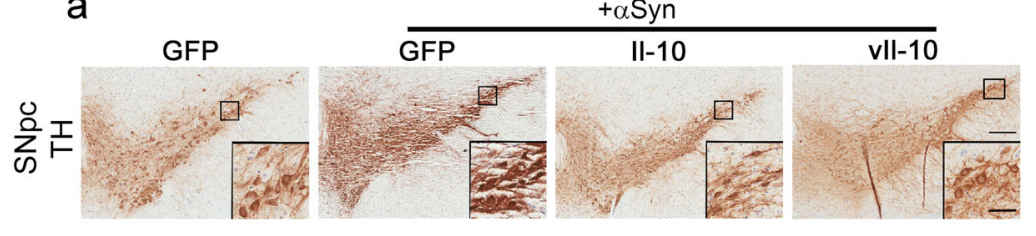

C
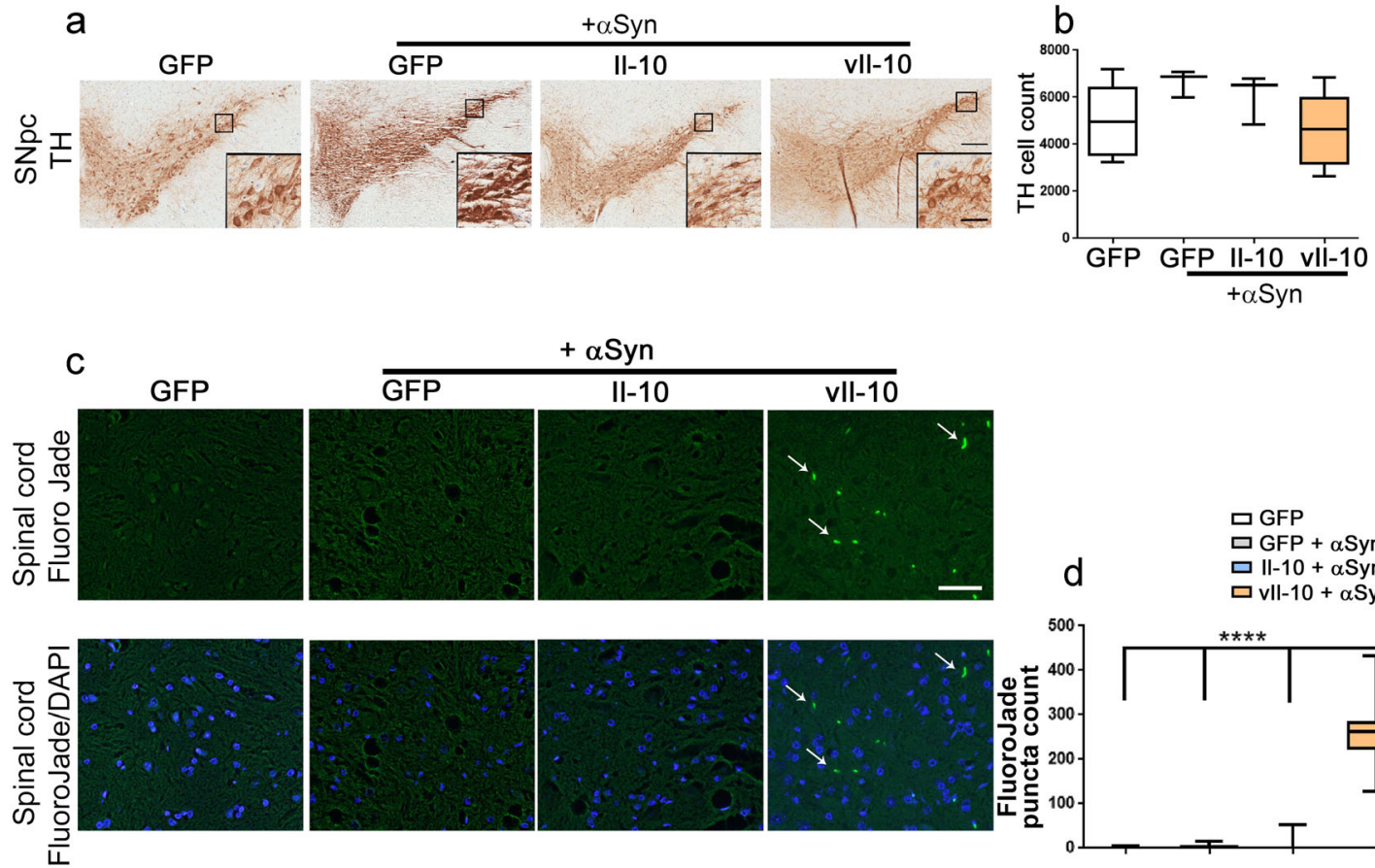
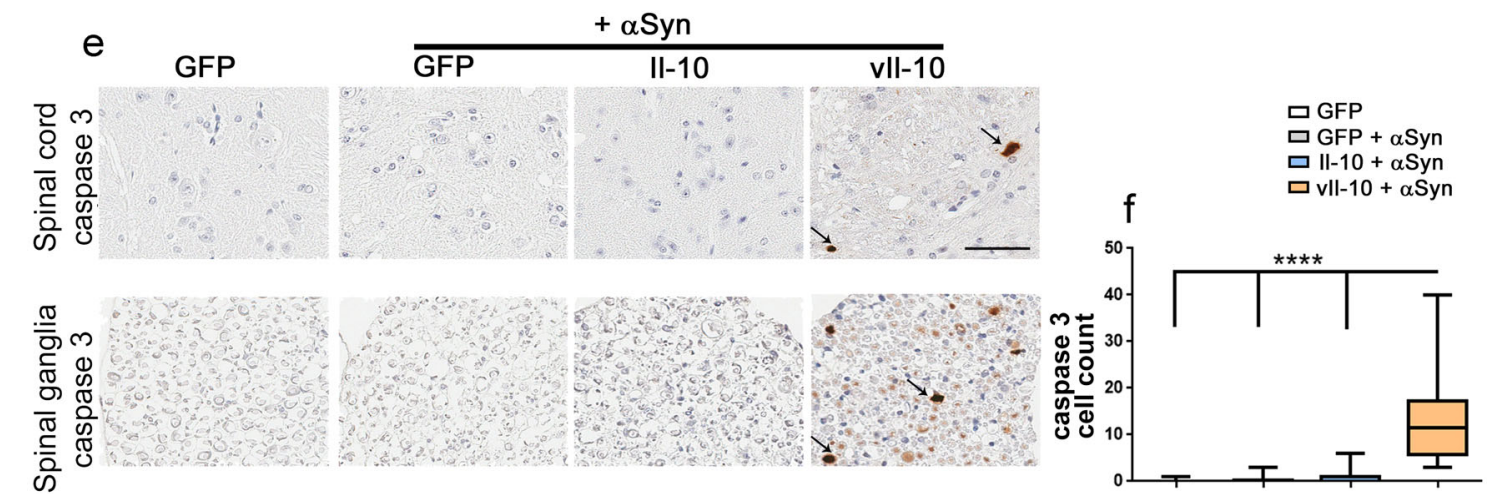

Fig. 6 Cell death analysis in aSyn aggregate-seeded hemizygous M83 + I- mice. a, b Midbrains of vehicle-injected AAV-GFP-expressing M83 $+/-$ mice or $\alpha$ Syn aggregate-seeded M83+/- mice expressing AAV-GFP, AAV-II-10, or AAV-vll-10 were stained for tyrosine hydroxylase (TH) (a), and the number of TH-positive dopaminergic neuronal cell bodies was manually counted (b). Scale bar, $200 \mu \mathrm{m}$ (main panel), $50 \mu \mathrm{m}$ (inset). $n=3-6$ mice/group; one-way ANOVA. c-f Cell death was analyzed in the lumbar spinal cord of M83+/- mice. Punctate Fluoro-Jade C staining was observed in the vIL-10-expressing $\alpha$ Syn-seeded M83+/- mice indicated by arrows (c, top panel). The bottom panel depicts a dual-color image incorporating the nuclear stain DAPI. The number of Fluoro-Jade C-labeled puncta was manually counted (d). Anti cleaved caspase 3 antibody was used to stain the spinal cord (e, top panel) and accompanying spinal ganglia (e, bottom panel) of mice. The number of cleaved caspase 3-positive cells from the spinal cord and spinal ganglia (arrows, e) was manually counted (f). In the box plot, the whiskers extend from the minimum to maximum values, with the midline representing the median. Scale bar, $20 \mu \mathrm{m}$ (c), $50 \mu \mathrm{m}$ (e). $n=5-7$ mice/group. One-way ANOVA, ${ }^{* * * *} P<0.0001$

clearance in the seeded M83+/- mice. Because of previously reported data that IL-10 can inhibit autophagy ${ }^{28,29}$, we reasoned that this might explain mechanistically the effect of II-10-mediated worsening of aSyn proteinopathy. Using p62 as a marker of autophagic dysfunction ${ }^{30}$, we observed that aSyn seeding induced modest p62 accumulation in the spinal cords of GFP and II-10expressing mice (Fig. 7a-d) and the midbrain (Fig. 7e, f) compared to non-seeded genotype-matched and age-matched M83+/mice. vll-10, on the other hand, resulted in a profound upregulation of intracellular p62-immunoreactive inclusions in the thoracic and lumbar spinal cords (Fig. $7 \mathrm{a}-\mathrm{d} ; P<0.001$ relative to $I-10, P<0.0001$ relative to GFP) as well as in the midbrain (Fig. 7e, f; $P<0.05$ relative to II-10, $P<0.01$ relative to GFP) of aSyn-seeded mice.

To further confirm autophagic disturbance in these mice, we assessed the levels of microtubule-associated protein 1 light chain
$3 \beta$ (MAP1LC3B) $^{30}$ or LC3B, a key regulatory component of autophagic degradation pathway. Cytoplasmic and nuclear accumulation of LC3B indicates abnormalities in autophagosome assembly, which is widely used for the detection of dysfunctional autophagy ${ }^{31}$. Anti MAP1LC3B staining revealed sparse staining in the thoracic and lumbar spinal cords of II-10-expressing aSynseeded mice (Fig. 7g-j) but not in the midbrain (Fig. 7l). On the other hand, vll-10 expression led to nuclear staining and diffuse cytoplasmic staining suggestive of accumulation of autophagic debris. Compared to GFP-expressing mice seeded with aSyn, vll10 mice showed increased LC3B levels in the thoracic and lumbar segments of spinal cords (Fig. $7 \mathrm{~g}-\mathrm{j} ; P<0.001$ in the thoracic spinal cord, $P<0.05$ in the lumbar spinal cord), whereas there was a trend toward increased LC3B staining in the midbrains (Fig. 7k, l; $P=0.22$ relative to control aSyn-seeded mice). To identify which 

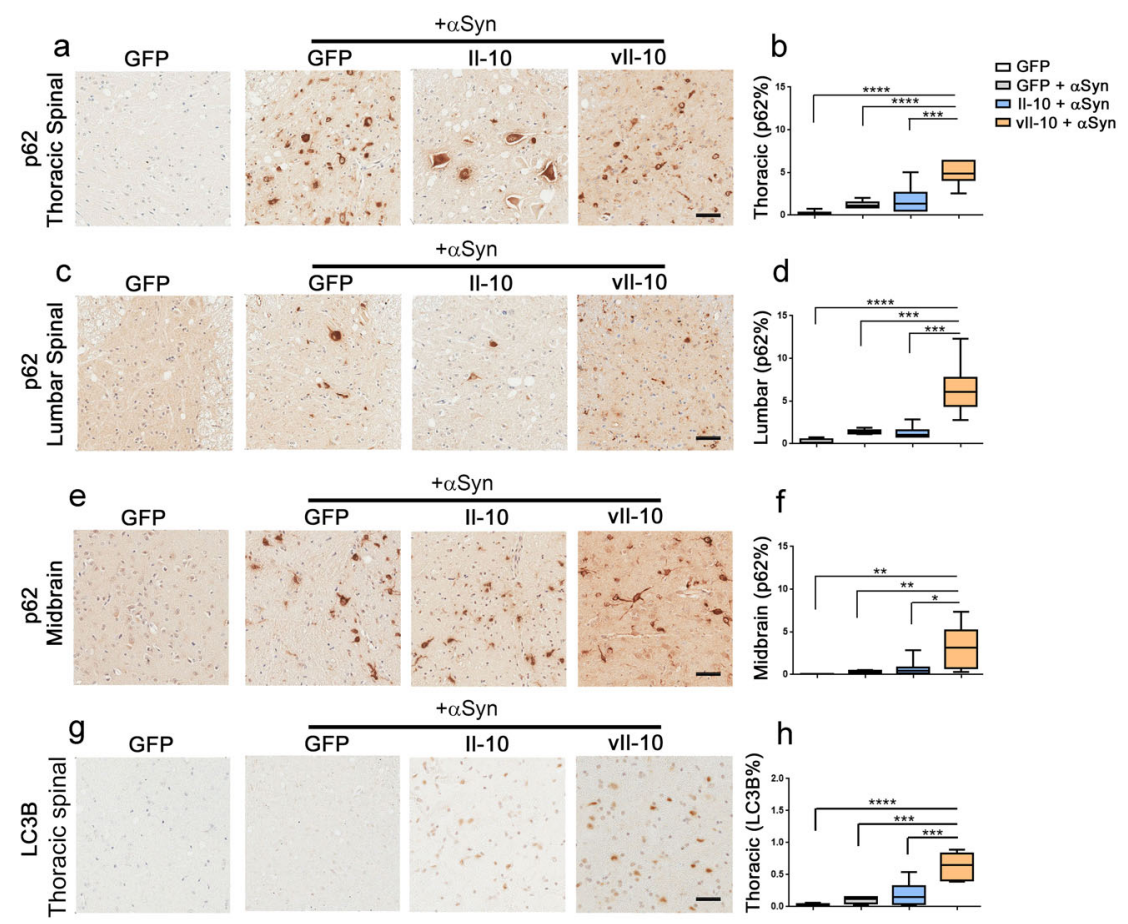

vll-10
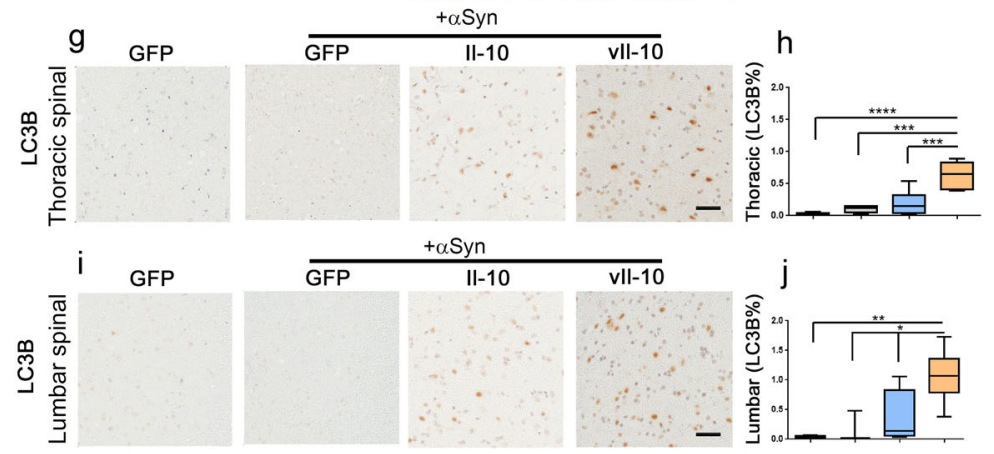

$+\alpha$ Syn

vll-10
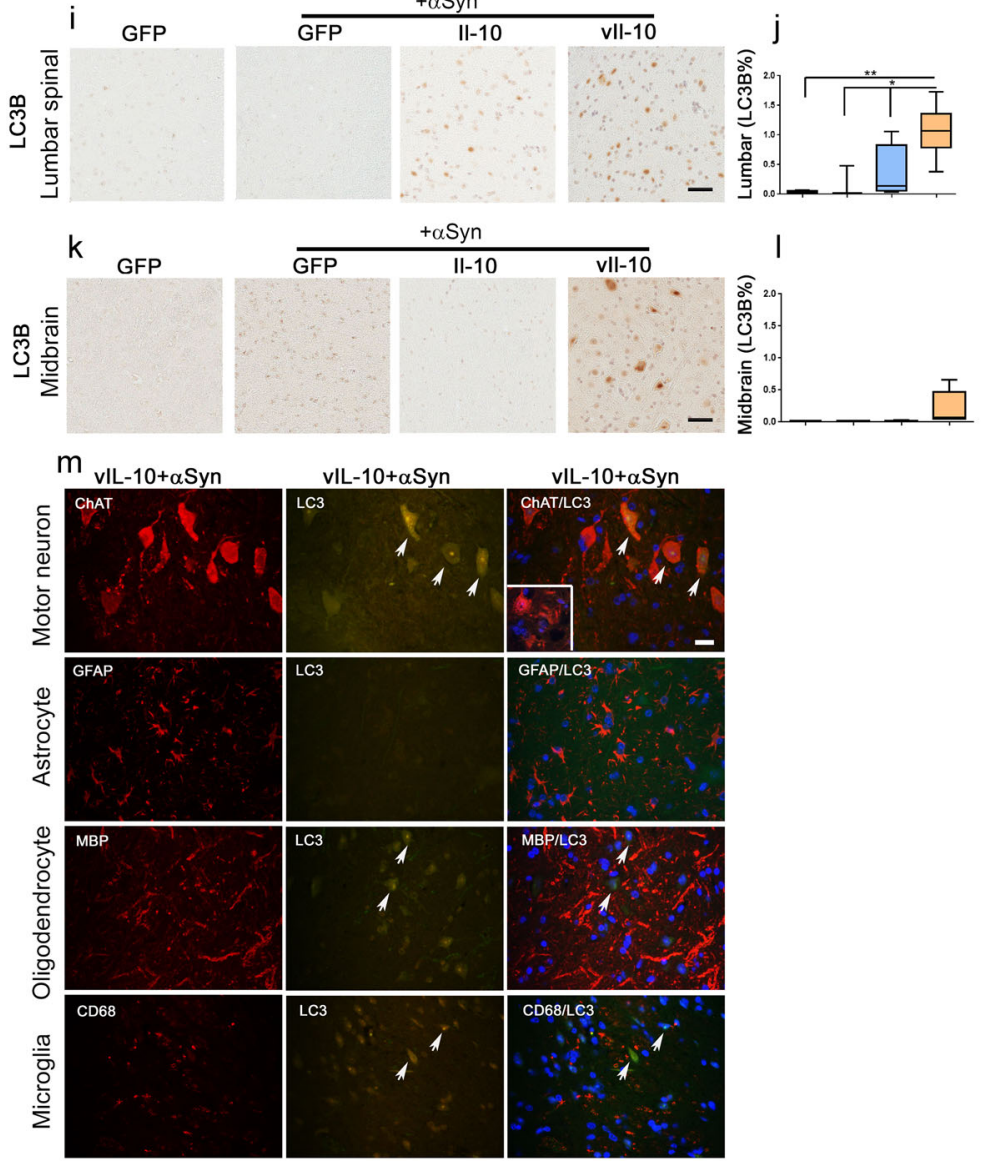

vIL-10+ + Syn
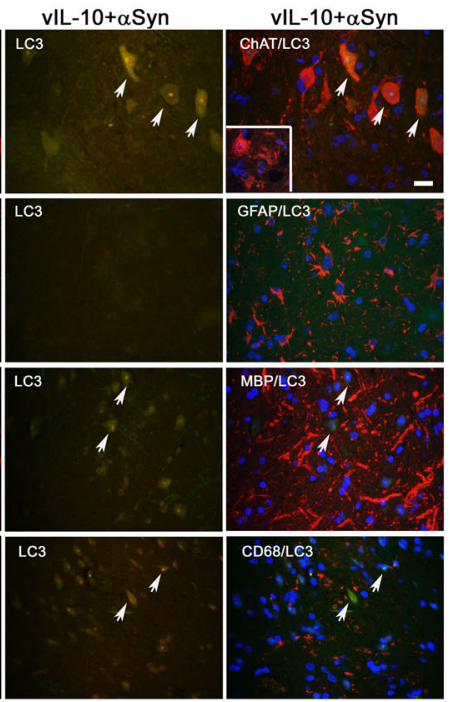

cells preferentially accumulated LC3B in response to vll-10, we performed a co-immunofluorescence analysis localizing LC3B in motor neurons (stained with choline acetyltransferase, ChAT), astrocytes (stained with GFAP), oligodendrocytes (stained with myelin basic protein, MBP), and microglia (stained with CD68).
Representative images from the lumbar spinal cord of vlL-10expressing mice seeded with aSyn fibrils showed robust LC3B staining in the ChAT-immunopositive neurons (arrows, top panel, Fig. $7 \mathrm{~m}$ ). None of the other cell types tested (astrocytes, oligodendrocytes, and microglia) show appreciable LC3B 
Fig. 7 Accumulation of autophagic markers in vll-10-expressing aSyn fibril-seeded hemizygous M83 $+I-$ mice. Neonatal M83 $+/-$ mice were injected with AAV-GFP, II-10, or vll-10 and were aged to 2 months when they were injected with $\alpha$ Syn fibrils in the muscle. PBS (vehicle) injection was the control for $\alpha$ Syn fibril injection and is represented by the immunohistochemistry panel on the left of each panel. Representative images of anti-p62/sequestosome antibody-stained tissue and immunostaining analysis (\% immunoreactivity burden) from the thoracic spinal cord (a, b), lumbar spinal cord (c, d), and midbrain $(\mathbf{e}, \mathbf{f})$ are shown. Representative images from MAP1LC3B antibodystained tissue and immunostaining analysis (\% immunoreactivity burden) derived from the thoracic spinal cord ( $\mathbf{g}, \mathbf{h})$, lumbar spinal cord (i, $\mathbf{j})$, and midbrain (k, I) are shown. Of note, $2 / 5$ mice in the II-10-expressing seeded mice showed LC3B staining in the spinal cord. $n=5-7$ mice/ group; one-way ANOVA; ${ }^{* * *} P<0.0001 ;{ }^{* * *} P<0.001 ;{ }^{*} P<0.01 ;{ }^{*} P<0.05$. Scale bar, $50 \mu \mathrm{m}$. In the box plot, the whiskers extend from the minimum to maximum values, with the midline representing the median. $\mathbf{m}$ Representative images from the lumbar spinal cord showing robust localization of LC3B (arrows, middle panels) in ChAT-immunopositive neurons (arrows, top panel) but not in GFAP-immunopositive astrocytes, MBP-immunopositive oligodendrocytes, or CD68-immunopositive microglia of vll-10-expressing Line M83+/- mice seeded with $\alpha$ Syn fibril. The right panels show merged images of LC3B (detected with Alexa fluor $488 \mathrm{~nm}$ ) with cell type-specific markers (detected with Alexa fluor $594 \mathrm{~nm}$ ). Slides were counterstained with DAPI. Inset in ChAT panel (right) denotes triple-color merged image from M83+/- mice seeded with $\alpha$ Syn fibril showing no detectable LC3B. Arrows (denoting LC3B signal) in MBP- and CD68-stained panels do not colocalize with oligodendrocyte or microglia, respectively. Scale bar, $10 \mu \mathrm{m}$.

colocalizing with the cell type-specific markers (Fig. $7 \mathrm{~m}$ ). Together, these observations indicate that preconditioning the neuraxis with II-10-related immunosuppressive signaling can lead to exacerbation of aSyn proteinopathy via altering neuronal autophagic pathways.

\section{DISCUSSION}

Gliosis is a cardinal feature in post mortem brains of PD patients ${ }^{32}$ as well as a prodromal feature in the early-stage PD patients and DLB patients ${ }^{33,34}$. Several cytokines with stimulatory propertiesfor example, IL- 6 and TNF- $a$-are increased in PD patient sera and are correlated with the severity of the nonmotor symptoms in PD patients ${ }^{35}$. Recent genome-wide association studies have also uncovered genetic overlaps in PD patients and patients suffering from autoimmune disorders ${ }^{36}$. In mouse models of synucleinopathy, we, as well as other groups, have reported robust inflammation in the end-stage mice ${ }^{15,37}$, and treatment with immunomodulatory agents prevented aSyn pathology ${ }^{38}$. These lines of evidence generally point to inflammatory signaling as a possible etiological factor in synucleinopathies. Our study was designed to test whether II-10 overexpression initiated before the onset of neuropathology or phenotypes would be able to suppress inflammation and ameliorate aSyn pathologies in the M83 model. Our approach in this study was to use recombinant AAVs to express II-10 based on its future translational potential as a vector for gene therapy ${ }^{39}$. Further, the AAV gene targeting approach allowed us to restrict II-10 expression to the CNS, thereby limiting its effect only to the target organ and minimizing off-target effects on peripheral immunity. Contrary to our expectations, we uncovered a detrimental effect of intraspinal II10 expression in our synucleinopathy models. We specifically found that II-10 overexpression in the spinal cord of homozygous $\mathrm{M} 83+/+$ mice leads to gliosis, moribund condition, and early death. In the second model where hemizygous M83+/- mice were seeded peripherally with preformed aSyn fibrils, we found that II-10 expression similarly led to reduced lifespan. In both cases, II-10-mediated accelerated death was not accompanied by increased aSyn inclusion pathology in mice phenotypically matched for paralysis with control cohorts, revealing an interesting dichotomy between neurodegenerative cascade and proteinopathy in the context of neuroinflammation. Previously, we have reported that AAV-II-10 worsened Alzheimer's disease (AD) pathology without affecting lifespan ${ }^{19}$. Intracranial Il-10 expression in this model worsened amyloid $\beta$ pathology by reducing microglial phagocytic activity and increasing ApoE sequestration within the extracellular amyloid $\beta$ plaques ${ }^{19}$. On the other hand, intraspinal AAV-II-10 (the same construct used in this study and ref. ${ }^{19}$ ) expression delayed paralysis and increased lifespan in the SOD1 model of ALS through moderating inflammatory chemokine signaling pathways ${ }^{18}$. Thus, the phenotypic and neuropathologic outcomes of II-10 signaling were widely disparate in these different neurodegenerative models, in spite of the fact that these experiments were performed on similar genetic backgrounds of mixed C57Bl6 lineage. Taken together, these observations serve as exemplars of the immunoproteostasis paradigm, whereby the same immune mediator can differentially influence the pathologic outcome based on the type of neurodegenerative proteinopathy and the context of the proteinopathy ${ }^{40}$.

To disentangle the pleiotropic actions of II-10 in the aSyn fibrilseeded M83+/- mouse model, we used a naturally occurring variant vll-10 that has predominantly immunosuppressive properties $^{16}$. Contrary to our expectation that this vll-10 would ameliorate synucleinopathy, we observed dramatic upregulation of gliosis, accumulation of soluble aSyn and pathological aSyn aggregates, and neuronal-predominant autophagic impairment in vll-10-expressing mice compared to control mice. This unexpected effect on autophagy impairment is consistent with previous observations that II-10 can impair lymphocyte- and dendritic cellmediated autophagy ${ }^{28,29}$. Interestingly, while vll-10 and II-10 both reduced lifespan in aSyn-seeded M83 mice, only vll-10 exacerbated intracellular and parenchymal aSyn accumulation. Though our data primarily suggests that the injurious outcome of vll-10 signaling in the aSyn model is mediated by neuronal autophagic impairment and a failure to clear neuronal aSyn, it is also possible that this phenotype can be influenced partly by vll-10-induced microgliosis. Taken together, our study points to a novel aspect of immune signaling in synucleinopathies, whereby II-10 conditioning can exacerbate the pathologic landscape through a combination of cell-autonomous and non-cell-autonomous means, as shown in other models of neurodegenerative diseases ${ }^{41}$.

Our study opens up an intriguing question surrounding the relative roles of immune cells in the aSyn aggregate-seeded model. Extensive immunohistochemical examination of microglial and astrocytic proliferation showed that, in spite of having predominantly immunosuppressive properties in the peripheral immune system ${ }^{16,17}$, vll-10 robustly upregulated microglial number in the spinal cord and midbrain of aSyn-seeded mice coinciding with increased intracellular aSyn inclusions and parenchymal aSyn levels. First, this would suggest that preconditioning the CNS of a neurodegenerative proteinopathy model with a cytokine having immunosuppressive properties does not necessarily dampen gliosis. Second, it is tempting to suggest that chronic immunosuppression can result in reduced microglial clearance of aSyn leading to intracellular pathologies, which then leads to increased microgliosis as a form of non-cell-autonomous response to the escalating proteinopathy. This would be consistent with our earlier report where we found that inflammatory preconditioning of the CNS by Interleukin- 6 restricted the seeding and transmission of aSyn in the brain ${ }^{42}$. Such non-cellautonomous effects of immune cells on neuronal proteinopathy is especially apparent in mouse models of $\mathrm{ALS}^{41}$. It is noteworthy 
that II-10 itself did not increase microgliosis in the brain or spinal cord or promoted synucleinopathy in the seeded model. Interestingly, neither II-10 nor vll-10 triggered astrogliosis in the spinal cord relative to GFP-expressing control mice. This highlights a clear disconnect between how microglia and astrocytes respond to the immune stimulus in different areas of the neuraxis. Such differential proliferation properties of microglia and astrocytes in response to vll-10 and II-10 underscores the complex role of immune signaling in neurodegenerative cascades. Future work based on spatial profiling techniques or single-cell-based techniques could clarify how microglial and astrocytic functions are differentially regulated in a spatial-temporal context and contribute to the pathologic trajectory of synucleinopathies.

Given the rich dataset implicating chronic neuroinflammation in $P D^{11}$, our data seem somewhat counter-intuitive. To illustrate this point, our data do not agree with at least two earlier studies where IL-10 showed a beneficial effect in acute toxin models of PD ${ }^{43,44}$. In the first study, human IL-10 infusion was shown to reverse lipopolysaccharide-mediated dopaminergic neurodegeneration ${ }^{43}$, and in the second study, AAV-IL-10 rescued toxin-induced reductions in dopamine levels ${ }^{44}$. Unlike the experimental models used in our study, these acute toxin models of PD used in these previous studies do not capture the gradual physiological process that is inherent in neurodegenerative synucleinopathies-thus, any direct comparisons would necessitate cautious interpretation. These acute toxin models also cause both neurodegeneration and neuroinflammation that are relatively nonspecific in nature in that neither of these models robustly recapitulate PD-type protein aggregation and extranigral pathology. In addition, because of their fast trajectory, these models cannot fully recapitulate the insidious nature of synucleinopathies as observed in our peripherally seeded M83+/- model. Using II-10 infusion is also ineffective as a therapy, as IL-10 has an extremely short half-life (2.0-4.5 h) that can severely limit the efficacy of the experimental paradigm $^{45}$. Taken together, it would seem that the outcomes of II-10 signaling in mouse models that recapitulate PD-relevant proteinopathy as reported in our study are physiologically distinct from the acute toxin models of dopaminergic neurodegeneration.

In conclusion, we have revealed an unexpected detrimental outcome following the expression of II-10 and vll-10 in mouse models of synucleinopathy. While the adverse effects of II-10 occurred without exacerbation of aSyn pathology, expression of vll-10 shortened lifespan via microgliosis, autophagic impairment, and accumulation of aSyn in aSyn-seeded mice. Our data demonstrate that immunosuppressive conditioning of the neuraxis exacerbates the pathologic landscape by altering intracellular protein-quality maintenance in conjunction with dysfunctional microgliosis.

\section{METHODS}

\section{Animals}

All animals were treated ethically as per protocols approved by the University of Florida Institutional Animal Care and Use Committee regulatory policies. Animals were maintained in specific pathogen-free conditions on a 12-h circadian cycle with unlimited access to food and water. Experiments were performed using standard blinding protocols of NC3R. Homozygous Line M83+/+ (Fig. 1) and hemizygous Line M83+/(Figs. 2-7) express the human aSyn (SNCA) gene with the A53T mutation under control of the mouse prion protein promoter ${ }^{13}$. Intramuscular (IM) injection of preformed aSyn fibrils in M83+/- mice lead to induction of aSyn inclusions and paralysis within $\sim 4$ months of fibril injection ${ }^{14,15}$ (Figs. 2-7). Mice used for all analyses were phenotype-matched for paralysis and moribund condition.

\section{aSyn fibril preparation and IM injection}

Recombinant mouse aSyn was expressed in E. coli and purified using sizeexclusion and ion-exchange chromatography, as previously described ${ }^{15}$. In total, $5 \mathrm{mg} / \mathrm{ml}$ mouse aSyn protein solubilized in sterile PBS (Invitrogen) was incubated at $37^{\circ} \mathrm{C}$ with continuous shaking at $1050 \mathrm{rpm}$. aSyn fibril formation was validated using K114 fluorometry, as previously described ${ }^{15}$. Immediately before injection, mouse aSyn fibrils were diluted to $1 \mathrm{mg} / \mathrm{ml}$ in sterile PBS and fragmented by water bath sonication for $1 \mathrm{~h}^{15}$. Twomonth-old M83 +/- mice were anesthetized with isoflurane. After shaving the back of the hindlimb, a 10- $\mu$ l Hamilton syringe with a 27-gauge needle was inserted $\sim 1 \mathrm{~mm}$ into the gastrocnemius muscle to deliver $5 \mu \mathrm{g}$ of aSyn fibril or $5 \mu$ l of sterile PBS in each hindlimb ${ }^{15}$.

\section{AAV preparation and injection}

GFP and murine II-10-expressing recombinant AAVs plasmids have been generated previously and described earlier ${ }^{18,19}$. 187A vll-10 was a kind gift from Dr. Scott Loiler and Dr. Terrence Flotte at the University of Florida. AAV serotype 1 was packaged by methods described earlier ${ }^{18,19}$. Briefly, AAV vectors expressing GFP, II-10 and, vll-10 under the control of the cytomegalovirus enhancer/chicken beta-actin (CBA) promoter, a woodchuck hepatitis virus post-transcriptional regulatory element (WPRE), and the bovine growth hormone polyA were transfected in HEK293T cells using linear polyethylenimine (PEl, Polysciences). Cells were co-transfected with the helper plasmid pDP1rs. After 4 days, the packaged virus was purified from the cell lysates using a discontinuous lodixanol gradient followed by buffer exchange in sterile PBS. The genomic titer was determined by quantitative PCR as described earlier ${ }^{19}$. AAVs were then aliquoted and stored at $-80^{\circ} \mathrm{C}$ until further use. For neonatal injections, AAV was diluted in sterile $1 \times$ DPBS, pH 7.2 to $1 \mathrm{E} 13$ vector genomes per $\mathrm{ml}$, and used immediately as described earlier ${ }^{18}$. Briefly, neonatal mice were cryoanesthetized for 3-4 min, resulting in the body temperature being lowered to $<10^{\circ} \mathrm{C}$ and injected with AAV using $10-\mu$ syringes ( 1 inch, 33 gauge, 30 degrees beveled needle; Hamilton Company). In total, $1 \mu$ of AAV was slowly injected into the midline, which can be seen as a white line down their back, about $5 \mathrm{~mm}$ from the base of the tail. Injected pups were allowed to recover on a heated pad and returned to their home cage.

\section{Tissue processing and immunohistochemistry}

Mice were euthanized with $\mathrm{CO}_{2}$ inhalation as per humane conditions and subsequently perfused using ice-cold saline containing heparin. Each spinal cord (cervical, thoracic, and lumbar segments) was divided into three sections $-12 \mathrm{~mm}$ from the proximal section (containing cervical and thoracic segments, referred to as "thoracic" henceforth), $4 \mathrm{~mm}$ from the midline (containing thoracic segment), and $12 \mathrm{~mm}$ from the distal section containing lumbar segment (referred to as "lumbar" henceforth). The proximal and distal sections were fixed in 10\% normal buffered formalin, while the midline section was flash-frozen for RNA analysis or biochemical analysis. Following paraffin processing, spinal cord fragments representing the thoracic and lumbar sections of each mouse were each serially dissected every $\sim 2 \mathrm{~mm}$ into three to four coronal sections and embedded in a paraffin block. Each paraffin block representing the thoracic or lumbar spinal cord was cut at $5 \mu \mathrm{m}$ thickness and used for immunohistochemistry. The entire brain was fixed in 10\% normal buffered formalin. Each brain was then sectioned coronally distanced at $4 \mathrm{~mm}$ using a brain matrix and processed for paraffin embedding. Thus, each brain slide contained four coronal sections representative of various anatomical levels of the whole brain. The brain paraffin blocks were sectioned at $10 \mu \mathrm{m}$ thickness. Paraffin-embedded tissues were immunostained using primary antibodies (Supplementary Table 10) followed by detection with ImmPRESS Polymer Reagents (Vector Laboratories) and visualized using 3,3'-diaminobenzidine (Vector Laboratories). Briefly, slides were deparaffinized, subjected to antigen retrieval, treated with $3 \%$ hydrogen peroxide, and blocked in $2 \%$ FBS prepared in $0.1 \mathrm{M}$ Tris buffer $(\mathrm{pH}$ 7.6) in preparation for primary antibody incubation overnight at $4{ }^{\circ} \mathrm{C}$. Following secondary antibody incubation on the subsequent day, the sections were counterstained with Mayer's hematoxylin (Sigma), dehydrated, and mounted in Cytoseal 60 (Fisher Scientific). Stained slides were scanned using a whole-slide imager (Aperio ScanScope CS, Leica Biosystems) and images visualized using the Aperio ImageScope software (Leica Biosystems). Quantification of immunostaining was done using the Positive Pixel count program configured to detect brown color (ImageScope, Aperio Technologies). The total number of brown pixels (positive) divided by the total number of pixels (positive +negative) was represented as a $\%$ immunoreactivity burden. For the spinal cord, the $\%$ immunoreactivity values on all serial sections on each slide were counted for any given sample and averaged for the cohort. For brain sections, the immunoreactivity value from bilateral midbrain or 
cortex areas were tabulated. For cleaved caspase 3, the total number of positive cells was counted as the staining pattern was sparse. The total number of cells on each slide were counted for any given sample and averaged for the cohort. For co-immunofluorescence staining, the method used was identical as described above, except that slides were not incubated in hydrogen peroxide, Alexa Fluor-conjugated secondary antibodies (1:500; Invitrogen) were used to detect signal, and slides were counterstained and mounted with 4',6-diamidino-2-phenylindole (DAPI; Southern Biotech). Images were captured using an Olympus BX60 Microscope fitted with an Olympus DP71 camera. Analyses were done on phenotype-matched mice. For the M83+/+ cohort, II-10-expressing mice typically were 4-6 months old and controls were typically 8-12 months old (Fig. 1), whereas for aSyn-seeded M83+/- cohort, endstage paralyzed mice typically aged 5-7 months were used (Figs. 2-7).

\section{Biochemical analysis of the injected spinal cord}

Biochemical analysis was done using the thoracic spinal cord segment. The frozen tissue was cryopulverized in liquid nitrogen and divided into aliquots for protein extraction or RNA extraction. The cryopulverized tissue was homogenized in RIPA buffer containing protease and phosphatase inhibitors (Pierce), and homogenates were cleared by centrifugation at $22,000 \mathrm{rpm}$ at $4{ }^{\circ} \mathrm{C}$ for $30 \mathrm{~min}$ (TLA-55 rotor, Beckman Coulter). II- 10 and vil10 levels were analyzed using RIPA-solubilized protein lysates using mouse-specific BD OptiEIA kits (BD Biosciences) as per the manufacturer's recommendations. GFAP immunoblots were done using RIPA-solubilized lysates using anti-rabbit GFAP antibody (1:500, Dako) as previously described $^{19}$. All blots derive from the same experiment and processed in parallel.

\section{Quantification of TH neurons in the substantia nigra (SN)}

Quantification of TH neurons were done based on a previously published protocol $^{46}$. Dopaminergic neurons stained with the anti-TH polyclonal antibody (Millipore, 1:1000) were counted manually. Serial sections were cut at $10 \mu \mathrm{m}$ intervals and slides containing the SN were delineated. Every 10th section was used for manual counting, whereby the number of TH neurons in the sections were averaged as representative of this particular area. The final count is a summation from each mouse and then averaged for each cohort.

\section{Fluoro-Jade C staining}

Paraffin-embedded slides were deparaffinized, rehydrated, and incubated at room temperature in $0.06 \% \mathrm{KMnO}_{4}$ solution for $10 \mathrm{~min}$. Slides were incubated in $0.0001 \%$ Fluoro-Jade C (EMD Millipore) dissolved in $0.1 \%$ acetic acid for $10 \mathrm{~min}$ with gentle shaking. Slides were washed, counterstained with DAPI, and dried on a slide warmer at $50{ }^{\circ} \mathrm{C}$ for $5 \mathrm{~min}$. Slides were cleared in xylene for $1 \mathrm{~min}$ and then mounted using Cytoseal 60 mounting medium (Fisher Scientific). Slides were imaged using an Olympus BX51 microscope mounted with a DP71 Olympus digital camera. One slide was stained per mouse (each slide contained 2-4 lumbar spinal cord coronal sections). The total number of Fluoro-Jade C-labeled puncta was manually counted for all the spinal cord sections of any one sample and then averaged for each cohort.

\section{RNA isolation and NanoString analysis}

RNA was extracted from cryopulverized aliquots of the thoracic spinal cords obtained from the midline section. For the M83+/+ group, all mice displayed moribund condition (average age of 3-4 months for II-10 group and 8 months for GFP group). For the preformed aSyn seed-injected M83 $+/$ - cohort, the analyses were done on the end-stage paralyzed mice (average age of 5-7 months). RNA was extracted, and $100 \mathrm{ng}$ of the total RNA was analyzed on a custom NanoString codeset as described earlier ${ }^{19}$. Raw count data for each sample was exported from RCC files using nSolver version 4.0. All samples passed QC analysis in nSolver. Count data were imported into $\mathrm{R}$ version 3.6 and Bioconductor version 3.9. Count matrices were normalized and differentially expressed genes were analyzed with DESeq2 version 1.24.0 using default normalization parameters and housekeeping genes included in the custom gene panel ${ }^{47}$. For gene ontology analysis, a false discovery rate (FDR) of less than 0.05 was used as a cutoff for significantly changed genes. Goseq version 1.36.0 was used for gene ontology analysis using the list of genes included on the custom NanoString array as background for analysis ${ }^{48}$. The $P$ values reported were adjusted for multiple comparisons (Padj). Graphs were generated with ggplot2 version 3.2.0. To calculate the M1-, M2-, and DAM-type signature, we used the geometric means of a set of genes previously identified to be increased under these specific conditions (Supplementary Table 2$)^{20}$. Geometric means, rather than arithmetic means, were used so as to reduce the effects of outliers which can skew the values. Briefly, the geometric mean of the counts for the selected genes within each list for each sample was calculated and then within the group, the mean and standard deviation of that value was calculated for the group score followed by a pairwise two-tailed $t$ test to test the significance of the association.

\section{Statistics}

For statistical comparisons, we used one-way ANOVA, multiple $t$ test with adjustments for multiple comparisons, or two-tailed $t$ test depending on the variables tested. Graphical data are plotted as box and whiskers plot, with the whiskers extending from the minimum to maximum values, with the midline representing the median. Graphical representation of data was done using Prism 6 (GraphPad Software, La Jolla, CA), and final images were created using Adobe Photoshop CC (Adobe Systems).

\section{Reporting summary}

Further information on research design is available in the Nature Research Reporting Summary linked to this article.

\section{DATA AVAILABILITY}

All data are contained in this paper and Supplementary information files. All materials used to generate the data will be available following the execution of the institutional material transfer agreement.

\section{CODE AVAILABILITY}

$\mathrm{R}$ Codes will be available on request from the corresponding author.

Received: 21 May 2020; Accepted: 28 January 2021; Published online: 19 March 2021

\section{REFERENCES}

1. Tan, E. K. et al. Parkinson disease and the immune system-associations, mechanisms and therapeutics. Nat. Rev. Neurol. 16, 303-318 (2020).

2. Doorn, K. J. et al. Microglial phenotypes and toll-like receptor 2 in the substantia nigra and hippocampus of incidental Lewy body disease cases and Parkinson's disease patients. Acta Neuropathol. Commun. 2, 90 (2014).

3. Hamza, T. H. et al. Common genetic variation in the HLA region is associated with late-onset sporadic Parkinson's disease. Nat. Genet 42, 781-785 (2010).

4. International Parkinson Disease Genomics, C. Imputation of sequence variants for identification of genetic risks for Parkinson's disease: a meta-analysis of genomewide association studies. Lancet 377, 641-649 (2011). et al.

5. Nalls, M. A. et al. Large-scale meta-analysis of genome-wide association data identifies six new risk loci for Parkinson's disease. Nat. Genet. 46, 989-993 (2014).

6. Lindestam Arlehamn, C. S. et al. Alpha-Synuclein-specific T cell reactivity is associated with preclinical and early Parkinson's disease. Nat. Commun. 11, 1875 (2020).

7. Sulzer, D. et al. T cells from patients with Parkinson's disease recognize alphasynuclein peptides. Nature 546, 656-661 (2017).

8. Kim, C. et al. Neuron-released oligomeric alpha-synuclein is an endogenous agonist of TLR2 for paracrine activation of microglia. Nat. Commun. 4, 1562 (2013).

9. Mao, X. et al. Pathological a-synuclein transmission initiated by binding lymphocyte-activation gene 3. Science 353, 6307 (2016).

10. Tang, D., Kang, R., Coyne, C. B., Zeh, H. J. \& Lotze, M. T. PAMPs and DAMPs: signal Os that spur autophagy and immunity. Immunol. Rev. 249, 158-175 (2012).

11. Hirsch, E. C. \& Standaert, D. G. Ten unsolved questions about neuroinflammation in Parkinson's Disease. Movement Disord. https://doi.org/10.1002/mds.28075 (2020).

12. Ouyang, W., Rutz, S., Crellin, N. K., Valdez, P. A. \& Hymowitz, S. G. Regulation and functions of the IL-10 family of cytokines in inflammation and disease. Annu. Rev. Immunol. 29, 71-109 (2011).

13. Giasson, B. I. et al. Neuronal alpha-synucleinopathy with severe movement disorder in mice expressing A53T human alpha-synuclein. Neuron 34, 521-533 (2002).

14. Sacino, A. N. et al. Intramuscular injection of alpha-synuclein induces CNS alphasynuclein pathology and a rapid-onset motor phenotype in transgenic mice. Proc. Natl Acad. Sci. USA 111, 10732-10737 (2014). 
15. Sorrentino, Z. A. et al. Motor neuron loss and neuroinflammation in a model of alpha-synuclein-induced neurodegeneration. Neurobiol. Dis. 120, 98-106 (2018).

16. Ding, Y., Qin, L., Kotenko, S. V., Pestka, S. \& Bromberg, J. S. A single amino acid determines the immunostimulatory activity of interleukin 10. J. Exp. Med. 191, 213-224 (2000).

17. Ouyang, P. et al. IL-10 encoded by viruses: a remarkable example of independent acquisition of a cellular gene by viruses and its subsequent evolution in the viral genome. J. Gen. Virol. 95, 245-262 (2014).

18. Ayers, J. I. et al. Widespread and efficient transduction of spinal cord and brain following neonatal AAV injection and potential disease modifying effect in ALS mice. Mol. Ther. 23, 53-62 (2015).

19. Chakrabarty, P. et al. IL-10 alters immunoproteostasis in APP mice, increasing plaque burden and worsening cognitive behavior. Neuron 85, 519-533 (2015).

20. Butovsky, O. \& Weiner, H. L. Microglial signatures and their role in health and disease. Nat. Rev. Neurosci. 19, 622-635 (2018).

21. Mosser, D. M. \& Zhang, X. Interleukin-10: new perspectives on an old cytokine. Immunol. Rev. 226, 205-218 (2008).

22. Groux, H. \& Cottrez, F. The complex role of interleukin-10 in autoimmunity. J. Autoimmun. 20, 281-285 (2003).

23. Santin, A. D. et al. Interleukin-10 increases Th1 cytokine production and cytotoxic potential in human papillomavirus-specific CD8(+) cytotoxic T lymphocytes. J. Virol. 74, 4729-4737 (2000).

24. Nemani, V. M. et al. Increased expression of alpha-synuclein reduces neurotransmitter release by inhibiting synaptic vesicle reclustering after endocytosis. Neuron 65, 66-79 (2010).

25. Farrer, M. et al. Comparison of kindreds with parkinsonism and alpha-synuclein genomic multiplications. Ann. Neurol. 55, 174-179 (2004).

26. Dhillon, J. S. et al. A novel panel of alpha-synuclein antibodies reveal distinctive staining profiles in synucleinopathies. PLOS ONE 12, e0184731 (2017).

27. Schmued, L. C., Stowers, C. C., Scalett, A. C. \& Xu, L. Fluoro-Jade C results in ultra high resolution and contrast labeling of degenerating neurons. Brain Res. 1035, 24-31 (2005).

28. Park, H. J. et al. IL-10 inhibits the starvation induced autophagy in macrophages via class I phosphatidylinositol 3-kinase (PI3K) pathway. Mol. Immunol. 48, 720-727 (2011).

29. Santarelli, R. et al. STAT3 activation by KSHV correlates with IL-10, IL-6 and IL-23 release and an autophagic block in dendritic cells. Sci. Rep. 4, 4241 (2014).

30. Deng, Z. et al. Autophagy receptors and neurodegenerative diseases. Trends Cell Biol. 27, 491-504 (2017).

31. Martinet, W., Roth, L. \& De Meyer, G. R. Y. Standard immunohistochemical assays to assess autophagy in mammalian tissue. Cells 6, https://doi.org/10.3390/cells6030017 (2017).

32. Croisier, E., Moran, L. B., Dexter, D. T., Pearce, R. K. \& Graeber, M. B. Microglial inflammation in the parkinsonian substantia nigra: relationship to alphasynuclein deposition. J. Neuroinflamm 2, 14 (2005).

33. Terada, T. et al. Extrastriatal spreading of microglial activation in Parkinson's disease: a positron emission tomography study. Ann. Nucl. Med. 30, 579-587 (2016).

34. lannaccone, S. et al. In vivo microglia activation in very early dementia with Lewy bodies, comparison with Parkinson's disease. Parkinsonism Relat. Dis. 19, 47-52 (2013).

35. Lindqvist, D. et al. Non-motor symptoms in patients with Parkinson's diseasecorrelations with inflammatory cytokines in serum. PLOS ONE 7, e47387 (2012).

36. Witoelar, A. et al. Genome-wide pleiotropy between Parkinson disease and autoimmune diseases. JAMA Neurol. 74, 780-792 (2017)

37. Liu, Z. et al. IL-17A exacerbates neuroinflammation and neurodegeneration by activating microglia in rodent models of Parkinson's disease. Brain Behav. Immun. 81, 630-645 (2019).

38. Gordon, R. et al. Inflammasome inhibition prevents alpha-synuclein pathology and dopaminergic neurodegeneration in mice. Sci. Transl. Med. 10, https://doi. org/10.1126/scitranslmed.aah4066 (2018)

39. Naso, M. F., Tomkowicz, B., Perry, W. L. 3rd \& Strohl, W. R. Adeno-associated virus (AAV) as a vector for gene therapy. BioDrugs 31, 317-334 (2017).

40. Golde, T. E. Harnessing immunoproteostasis to treat neurodegenerative disorders. Neuron 101, 1003-1015 (2019).

41. Chen, H., Kankel, M. W., Su, S. C., Han, S. W. S. \& Ofengeim, D. Exploring the genetics and non-cell autonomous mechanisms underlying ALS/FTLD. Cell Death Differ. 25, 648-662 (2018).
42. Koller, E. J., Brooks, M. M., Golde, T. E., Giasson, B. I. \& Chakrabarty, P. Inflammatory pre-conditioning restricts the seeded induction of alpha-synuclein pathology in wild type mice. Mol. Neurodegen. 12, 1 (2017).

43. Arimoto, $T$. et al. Interleukin-10 protects against inflammation-mediated degeneration of dopaminergic neurons in substantia nigra. Neurobiol. Aging 28, 894-906 (2007)

44. Joniec-Maciejak, I. et al. The influence of AAV2-mediated gene transfer of human IL-10 on neurodegeneration and immune response in a murine model of Parkinson's disease. Pharm. Rep. 66, 660-669 (2014).

45. Huhn, R. D. et al. Pharmacodynamics of subcutaneous recombinant human interleukin-10 in healthy volunteers. Clin. Pharm. Ther. 62, 171-180 (1997).

46. Kitada, T., Tong, Y., Gautier, C. A. \& Shen, J. Absence of nigral degeneration in aged parkin/DJ-1/PINK1 triple knockout mice. J. Neurochem. 111, 696-702 (2009).

47. Love, M. I., Huber, W. \& Anders, S. Moderated estimation of fold change and dispersion for RNA-seq data with DESeq2. Genome Biol. 15, 550 (2014).

48. Young, M. D., Wakefield, M. J., Smyth, G. K. \& Oshlack, A. Gene ontology analysis for RNA-seq: accounting for selection bias. Genome Biol. 11, R14 (2010).

\section{ACKNOWLEDGEMENTS}

This work was supported by NIH grant NS099738 (P.C.), the University of Florida Moonshot Initiative (P.C.), and Michael J Fox Foundation (T.E.G.).

\section{AUTHOR CONTRIBUTIONS}

S.G.C., E.J.K., M.M.T.B., E.G.D.L.C., and P.C. performed mouse experiments and data analysis; K.N.M. and C.C.D. collected NanoString data and performed analysis; P.E.C. generated recombinant AAV constructs; A.W.R. assisted in AAV production; T.E.G., B.I.G., and Y.R.L. assisted in initiation and development of the project; P.C. and D.R.B. participated in data interpretation and paper writing. All authors have read the paper.

\section{COMPETING INTERESTS}

The authors declare no competing interests.

\section{ADDITIONAL INFORMATION}

Supplementary information The online version contains supplementary material available at https://doi.org/10.1038/s41531-021-00169-8.

Correspondence and requests for materials should be addressed to P.C.

Reprints and permission information is available at http://www.nature.com/ reprints

Publisher's note Springer Nature remains neutral with regard to jurisdictional claims in published maps and institutional affiliations.

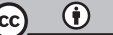

Open Access This article is licensed under a Creative Commons Attribution 4.0 International License, which permits use, sharing, adaptation, distribution and reproduction in any medium or format, as long as you give appropriate credit to the original author(s) and the source, provide a link to the Creative Commons license, and indicate if changes were made. The images or other third party material in this article are included in the article's Creative Commons license, unless indicated otherwise in a credit line to the material. If material is not included in the article's Creative Commons license and your intended use is not permitted by statutory regulation or exceeds the permitted use, you will need to obtain permission directly from the copyright holder. To view a copy of this license, visit http://creativecommons. org/licenses/by/4.0/.

(c) The Author(s) 2021 